\title{
Symmetry considerations for topology design in the elastic inverse homogenization problem
}

\author{
J.M. Podestá ${ }^{1}$, C. Méndez ${ }^{1}$, S. Toro ${ }^{1}$, A.E. Huespe ${ }^{1,2,3 *}$ \\ ${ }^{1}$ CIMEC-UNL-CONICET, Predio Conicet "Dr Alberto Cassano", CP 3000 Santa Fe, Argentina \\ ${ }^{2}$ Centre Internacional de Metodes Numerics en Enyinyeria (CIMNE),Campus Nord UPC. \\ ${ }^{3}$ E.T.S d'Enginyers de Camins, Canals i Ports, Technical University of Catalonia (Barcelona Tech) \\ Campus Nord UPC, Mòdul C-1, c/ Jordi Girona 1-3, 08034, Barcelona, Spain
}

Keywords: metamaterial design; elastic symmetry; topology optimization; inverse homogenization; tailored elastic properties.

\begin{abstract}
An analysis of the symmetries characterizing the micro-architecture topologies and the elastic material properties is performed. The goal is to elucidate a systematic procedure that facilitates the design of elastic metamaterial with a prescribed target elasticity tensor via inverse homogenization methodologies. This systematic procedure, which is defined through a set of rules, is based on the relationship established between the elasticity tensor symmetries and the symmetry displayed by the micro-architecture topology.

Following this procedure, it can be guaranteed that the designed composites, with the attained micro-structures, have effective elasticity tensors that possess the same or higher symmetries than those shown by the target elasticity tensors. Furthermore, the micro-architectures designed through this technique display simple topologies.

Both properties that are supplied by the procedure, i.e., the accomplishment of the required symmetry of the composite homogenized elasticity tensor combined with the topology simplicity, are assessed through numerical simulations of several micro-architecture design problems. They are designed by formulating the inverse homogenization problem as a topology optimization problem which is solved with two different standard algorithms. The proposed procedure and the conclusions here obtained do not depend on the algorithm adopted for solving this problem.
\end{abstract}

\footnotetext{
*Corresponding author. E-mail address: ahuespe@cimec.unl.edu.ar (A.E. Huespe).
} 


\section{Introduction}

The goal of the inverse homogenization problem addressed in this work is the microarchitecture design of a two-phase periodic elastic composite whose effective elasticity tensor $\boldsymbol{C}^{h}$ is identical to a target elasticity tensor $\hat{\boldsymbol{C}}$. In this context, a systematic procedure for restricting the search space of variables that are meaningful in this problem is described.

An approach to solve the mentioned inverse homogenization problem has been reported in the past by formulating it as a topology optimization problem, see for instance the landmark works by Sigmund ([1] and [2]) who has coined the name inverse homogenization to this kind of problem. See also the reference book by Bendsoe and Sigmund [3] and the bibliography cited therein related to this topic. According to this approach, the topology optimization problem is formulated by choosing a design domain, $\Omega_{\mu}$, assumed as a base cell of the composite, which is also used to compute its overall elastic properties. The algorithm solving the topology optimization problem tests different distributions of given hard and soft materials to satisfy a proposed optimality condition, see for example the methodologies proposed by Huang et al. [4], Amstutz et al. [5], Ferrer et al. [6], and [7] where the design of graded micro-architectures has been addressed.

The selection of the cell $\Omega_{\mu}$ is a decision that has to be taken by the designer and is a particular aspect of the inverse problem that we want to highlight in this work. There are several issues related to this choice. For example, Coelho et al. [8] investigate the cell size influence on the designed topology.

Also, Diaz and Benard [9] mention that the shape of the design domain plays an important role in widening the range of attainable micro-architectures with unusual properties. This has been the case for developing new classes of extreme material micro-structures by using rectangular unit cells and certain material distribution symmetries, see the cases studied in [10]. The relationship between tensor symmetry and unit cell shape has already been discussed by Barbarosie et al. [11] and Lukkassen et al. [12]. These authors exploit the symmetry of the material configuration within the unit cell to get a less expensive computation of the effective material properties. But, in these cases, the morphological symmetries have not been used to facilitate the search of a topology satisfying the inverse homogenization problem requirement.

In this work, we make an exhaustive analysis of the information associated with the target elasticity tensor. This information could be used to make easier the inverse design procedure. In particular, we aim to study the elasticity tensor symmetry and its connection with the base cell geometry and the material configuration within this cell. Then, based on this analysis, we introduce a set of guidelines, which can be taken as a protocol to guarantee the accomplishment of the required elastic symmetry. 
To reach this goal, arguments taken from crystallography are employed. Typically, each crystal is associated with a point group through its Bravais lattice and its motif. This association helps to determine the crystal system, as well as, the symmetry of any effective material property, according to Neumann's principle ([13]). See the discussion about the connection between material and physical symmetries reported by Zheng and Boehler [14].

In a complete parallelism, a Bravais lattice and a motif can be assigned to each periodic material configuration. Therefore, by following the same arguments to that given in crystallography, the material micro-architecture can be classified by possessing one of the seventeen plane groups with a given point group. Also, in this case, the point group of the material configuration geometry is connected with the effective elasticity tensor symmetry class. Hence, when this notion is applied to the inverse homogenization problem, the plane group characterization of the micro-structure could give a hint for distributing the material within the design domain such that the effective properties of the composite display a similar or higher symmetry than that shown by the target elasticity tensor. This is the principal line of argument taken by this work.

Another aspect related to the choice of the design domain has to be remarked. The symmetry properties of the target elasticity tensor give useful information to select the base cell shape between a family of cells. However, the slenderness of the adopted cell, taken from one particular family, should be defined by introducing more information. We propose to build a database storing effective elasticity tensors of composites with a set of representative and simple topologies. Then, the stored information in this database is used to choose the cell slenderness, as well as, to provide an initial configuration to solve the topology optimization algorithm.

A brief description of this paper is given as follows. Initially, we analyze the point and plane groups properties of different crystal systems in two-dimensions, as well as the point groups of the Bravais lattices associated with these crystals. We perform an exhaustive analysis of the full set of possible symmetries that can display a periodic crystal.

Next, we define a criterion to build the database. Based on this database and the symmetry notions previously discussed, in Section 4 we present the procedure through a series of rules that facilitates the inverse homogenization process.

The influence of these rules on the topology design process are assessed in Section 5 by means of a set of numerical simulations. We solve some representative inverse homogenization problems with techniques that follow the proposed rules and the solutions are compared with those obtained using conventional approaches that do not follow these rules. 


\subsection{Inverse material design as a topology optimization prob- lem}

Material design via inverse homogenization refers to the problem of finding the micro-architecture configuration of a composite whose effective elasticity tensor is identical to a target elasticity tensor. This problem involves two characteristic scale lengths; the macro-scale length, $\ell$, which is of the same order of magnitude as that of the structure size, and the micro-scale length, $\ell_{\mu}$, which is of the same order of magnitude as that of the material micro-architecture characteristic length. We assume that $\ell_{\mu} \ll \ell$. The effective elasticity tensor is defined at the macro-scale level, such as sketched in Figure 1, and the material design is performed at the scale $\ell_{\mu}$.

Material design via inverse homogenization has been formulated as a topology optimization problem in a given design domain and we follow a similar formulation in this paper.

Let us consider a structure whose material is a periodic composite constituted by two isotropic elastic phases $M_{1}$ and $M_{2}$. We take a basic micro-cell of this material identified by $\Omega_{\mu}$. In this micro-cell, phases $M_{1}$ and $M_{2}$ occupy the domains $\Omega_{\mu}^{1}$ and $\Omega_{\mu}^{2}$, respectively, see Figure 1 .

The characteristic function $\chi(\boldsymbol{y})$ in $\Omega_{\mu}$ identifies the positions where the phase $M_{1}$ is placed and is defined by:

$$
\chi(\boldsymbol{y})=\left\{\begin{array}{ll}
0 & \forall \boldsymbol{y} \in \Omega_{\mu}^{2} \\
1 & \forall \boldsymbol{y} \in \Omega_{\mu}^{1}
\end{array} .\right.
$$

Evidently, the homogenized elasticity tensor of the composite, $\boldsymbol{C}^{h}$, depends on the geometrical configuration of the phases $M_{1}$ and $M_{2}$ in $\Omega_{\mu}$. This dependence is made explicit by introducing the notation $\boldsymbol{C}^{h}(\chi)$. This tensor can be evaluated in $\Omega_{\mu}$ by enforcing periodic boundary conditions in displacements fluctuations. Then, standard computational techniques based on finite elements ([15], [16]) or Fast Fourier Transform ([17]) can be used to get this goal.

Next, we formulate the micro-architecture inverse design problem as a topology optimization problem expressed as follows: given the design domain $\Omega_{\mu}$ and the target effective elasticity tensor $\hat{\boldsymbol{C}}$, find the characteristic function $\chi$ satisfying:

$$
\begin{aligned}
& \min _{\chi} \frac{1}{\left|\Omega_{\mu}\right|} \int_{\Omega_{\mu}} \chi d \Omega \\
& \quad \text { such that: }\left\|\boldsymbol{C}^{h}(\chi)-\hat{\boldsymbol{C}}\right\|=0 .
\end{aligned}
$$

The cost function represents the stiff phase volume fraction. In particular, considering that the soft phase is void, the problem (2) identifies a minimum weight problem. 


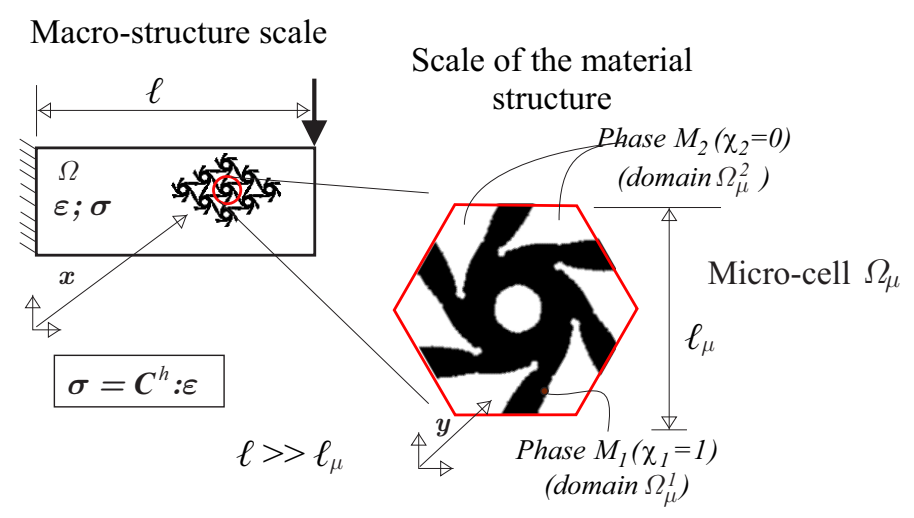

Figure 1: The material design is carried out by solving an inverse homogenization technique formulated as a topology optimization problem which involves two characteristic scale lengths. The micro-cell $\Omega_{\mu}$ is used as the design domain for the topology optimization problem. The symbols $\sigma$ and $\varepsilon$ represent the macro-stress and macro-strain tensors, respectively.

There are several implicit variables in the problem (2). One of particular interest here is the design domain, $\Omega_{\mu}$, where this problem is posed. The shape of this domain is a variable that should be fixed in advance; it results from a decision taken by the designer. Also, the enforcing of periodic boundary conditions along pre-established directions to find the effective properties of the composite, $\boldsymbol{C}^{h}$, is a decision taken in advance by arguing that the micro-architecture is periodic along these directions. Due to this arguments, the full material architecture must result from a spatial replica, by tessellation, of the cell $\Omega_{\mu}$.

We remark that both decisions play an important role to govern the complexity of the attained micro-architecture topology.

\section{Effective elastic symmetry inherited from the micro-architecture configuration}

Effective elastic properties of composites constituted by two isotropic phases show different classes of symmetries. These symmetries are a result of the micro-structure geometry, due to the spatial distribution of phases.

In this Section, by resorting to a complete parallelism with crystallography, we analyze the connection between the overall elastic properties and the micro-structure geometry, from the common perspective involving the symmetry properties.

First, we categorize the periodic material micro-architectures according to their point groups. To reach this goal, we identify the underlying Bravais lattice, the motif and the crystal system of the material configuration. This classification involves only the geometrical features of the composite. See the book of Sólyom [18] for additional information about this topic. Then, the elastic symmetry classes of these composites 
are briefly discussed and presented. Finally, we close this Section by discussing the connection between physical and material configuration symmetries stated in terms of Neumann's principle.

\section{Point group symmetry}

An isometric transformation imposed on the material configuration, which leaves invariant its spatial distribution, is a symmetry operation. The composition of successive symmetry operations is also a symmetry operation for that material configuration. So, from an algebraic point of view, this set of transformations constitutes a group. One simple operation of this group is a symmetry element.

First, we consider a restricted set of symmetry transformations which leaves a point of the material fixed. In this case, the symmetry elements are the rotations around a fixed axis (orthogonal to the plane of analysis), reflections across straight lines intersecting the fixed point and inversion in the fixed point. The last one can be ignored as it corresponds to a rotation through an angle of $\pi[\mathrm{rad}]$. The element of the group denoted $n$ is a rotation through an angle $2 \pi / n$ [rad] and the element denoted $m$ is a reflection through a plane.

Now, consider the groups that contain mirror lines and rotations. Any line

obtained from a mirror line via a rotation with the angle $2 \pi / n$ [rad], around the $n$-fold axis, is also a mirror line. Then:

- for $n$ odd, the angular separation of the $n$ mirror lines obtained in this way is $\pi / n$ [rad]. Thus, the group has $2 n$ symmetry elements, $n$ rotations and $n$ reflection planes, and is denoted by $\mathrm{nm}$;

- for $n$ even, rotations of a mirror line will yield only $n / 2$ different mirror lines whose angular separation is $2 \pi / n$ [rad]. Nevertheless, in this case, there must exist another set of mirror lines, i.e., the angle bisectors of the previously obtained lines. This is so because the composition of a reflection, in a mirror line, and a rotation through $2 \pi / n$ [rad] is equivalent to a reflection in a mirror line that makes an angle $\pi / n$ with the original mirror line. Therefore, there are two independent sets of mirror lines. This is expressed by the notation $n m m$ of such groups.

Considering the material distribution in the space, we can identify all the isometric transformation with fixed point leaving invariant this distribution. The set of all the symmetry elements of a given material is called its point group.

\subsection{Materials with periodic micro-architecture}

Let us consider materials having periodic micro-architectures with a given translational symmetry. The micro-architecture is invariant under discrete translations 
along two directions defined by the non-proportional primitive vectors $\boldsymbol{a}_{1}$ and $\boldsymbol{a}_{2}$. The translation of the material along directions that result from integer multiples of $\boldsymbol{a}_{1}$ and $\boldsymbol{a}_{2}$ takes the material into itself. Therefore, the characteristic function $\chi_{i}$, for a given $i$-th phase, satisfies

$$
\chi_{i}(\boldsymbol{x}+\boldsymbol{t})=\chi_{i}(\boldsymbol{x}) \quad ; \quad \text { where } \quad \boldsymbol{t}=\omega_{1} \boldsymbol{a}_{1}+\omega_{2} \boldsymbol{a}_{2}
$$

and the scaling factors $\omega_{1}$ and $\omega_{2}$ are two arbitrary integers.

\subsubsection{Underlying Bravais lattices of a periodic pattern}

Every periodic configuration of material possesses an underlying Bravais lattice and a motif. The procedure to identify the underlying lattice of a periodic structure and its motif is indicated in the artistic wallpapers reproduced in Figure 2. The lattice and the primitive vectors are found by identifying the equivalent set of points of the material periodic array. For example, equivalent sets of points are the eyes of the fishes in Figure 2-a and the red points in Figure 2-b. Once the Bravais lattice is characterized, the unit cells can be easily recognized. The motif is the material configuration pattern within a unit cell.

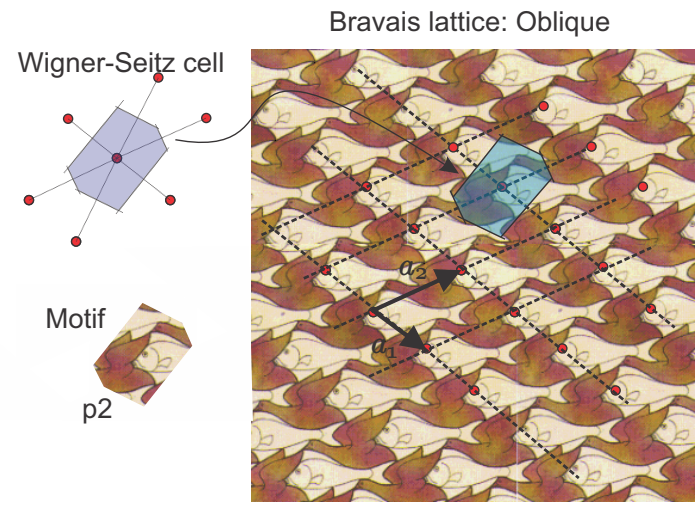

(a)

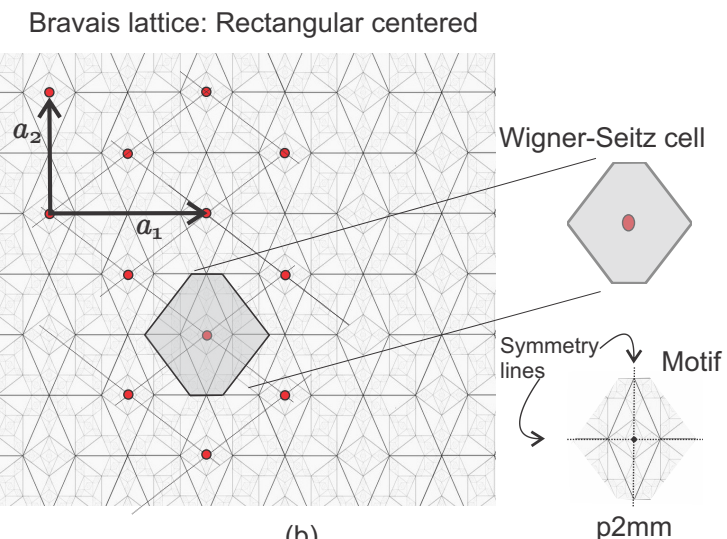

(b)

Figure 2: Identification of the underlying Bravais lattices, primitive vectors, Weigner-Seitz (Voronoi) cells and plane groups of artistic wallpapers by: (a) Escher; b) Geometrical draw.

According to the angle that the primitive vectors $\boldsymbol{a}_{1}$ and $\boldsymbol{a}_{2}$ form and the ratio between their magnitudes, only five different types of Bravais lattices can be identified. They are depicted in Figure 3 and are called: Hexagonal, Square, Rectangular primitive, Rectangular centered and Oblique.

\section{Lattice point group}

Due to the characteristic translational symmetry of Bravais lattices, and considering the crystallographic restriction theorem, there can only be a finite number of 
point groups for the five types of lattices. They are $n$-fold angles of symmetries, where $n \in\{2,3,4,6\}$, with one or two systems of symmetry lines. Then, the only possible point groups that Bravais lattices can have are the following four types: 2, $2 \mathrm{~mm}, 4 \mathrm{~mm}, 6 \mathrm{~mm}$. In this notation ${ }^{1}$, the number identifies the $n$-fold angle of rotational symmetry and $m$ and $m m$ means one or two mirror line systems. A mirror line system is the set of reflection lines which are obtained by the $n$-fold rotation of one mirror line.

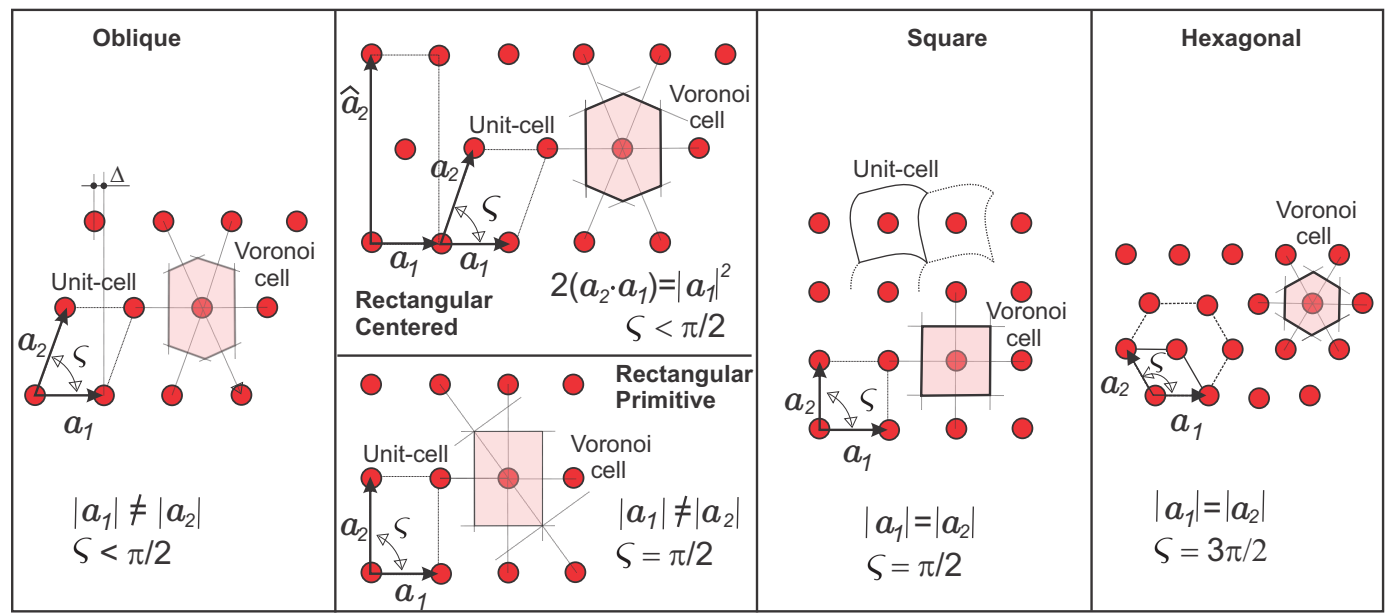

Figure 3: The five Bravais lattice types in the plane.

All the symmetry elements with a fixed point of the five types of plane lattices are shown in Figure 4. The fixed point can be any one of the lattice atoms. Therefore, the respective point group of each lattice can also be identified. They are shown in the same Figure.

The lattice system is formed by collecting the lattice types sharing similar point groups. So, due to the fact that the Rectangular primitive and the Rectangular centered lattices share the same point group, $2 \mathrm{~mm}$, they are grouped into one lattice system identified as the Rectangular lattice systems. Therefore, the four lattice systems are Oblique, Rectangular, Square and Hexagonal.

A Bravais lattice is the tessellation of a repetitive pattern, i.e., the juxtaposition of a given domain by the translation along the primitive directions. The domains with the repetitive patterns which have the smallest areas are the unit cells of the lattice, such as shown in Figure 4. In particular, we focus on the Weigner-Seitz ${ }^{2}$ (or Voronoi) unit-cell of the Bravais lattice.

\footnotetext{
${ }^{1}$ We use the International or Hermann-Mauguin notation, see [19].

${ }^{2}$ The Weigner-Seitz cell of a lattice is the domain centered in a lattice atom and comprising all the spatial points that are closer to the central atom than to any other atom of the lattice.
} 


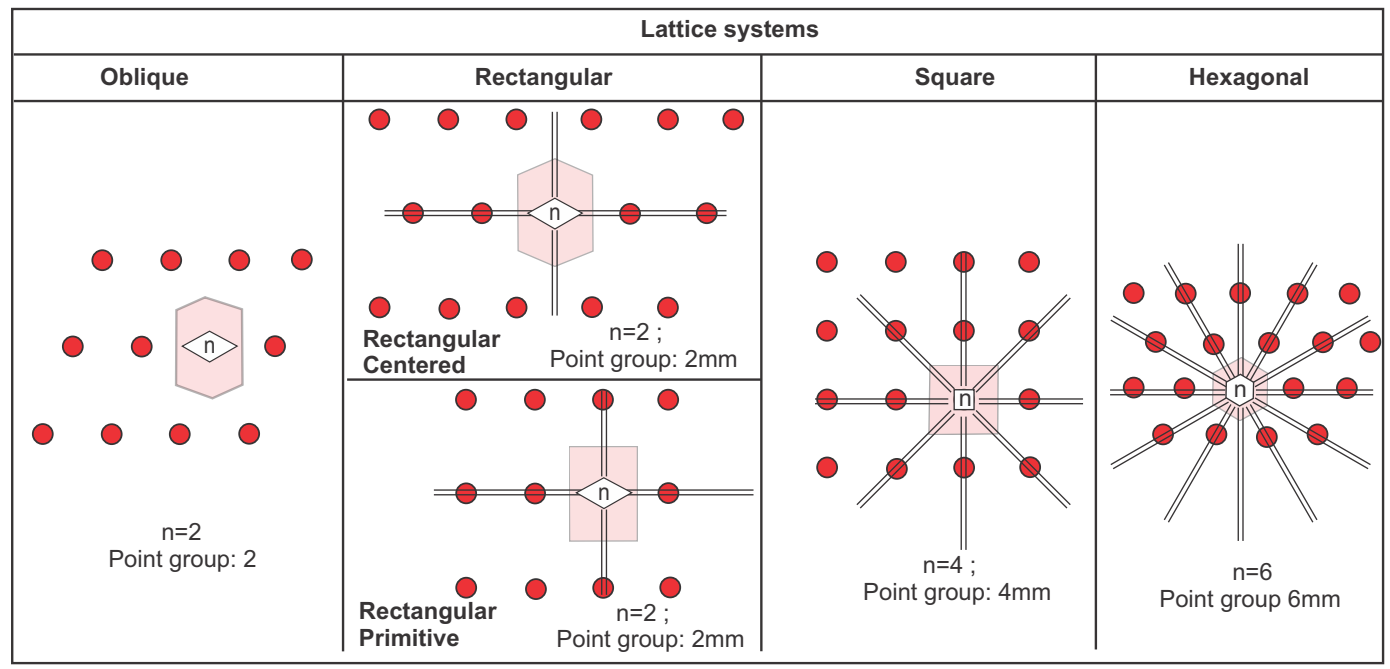

Figure 4: Rotational and reflection symmetries of Bravais lattices. Mirror planes (reflection symmetries) are depicted with two parallel lines; rotational symmetries are identified with the rotation angle $2 \pi / n$ around an axis perpendicular to the plane. Parameters $n$ and the lattice point groups are depicted. Voronoi cells have the same point groups as that shown by the associated lattices, i.e., they preserve the symmetries of the lattices. According to the lattice point group, there are four Bravais lattice systems: Oblique (2), Rectangular (2mm), Square (4mm) and Hexagonal $(6 \mathrm{~mm})$. The Rectangular system has two subsystems: centered and primitive.

Remark: the Weigner-Seitz cell and the associated lattice have the same point group. This statement is graphically observed in Figure 4.

\section{Parametrization of Bravais lattices}

All Bravais lattices can be characterized through the following two parameters:

$$
\begin{aligned}
\omega & =\left\|\boldsymbol{a}_{2}\right\| /\left\|\boldsymbol{a}_{1}\right\| ; \\
\varsigma & =\arccos \left[\left(\boldsymbol{a}_{2} \cdot \boldsymbol{a}_{1}\right) /\left(\left\|\boldsymbol{a}_{2}\right\|\left\|\boldsymbol{a}_{1}\right\|\right)\right] .
\end{aligned}
$$

Each pair of values $\omega, \varsigma$ defines a Bravais lattice. It can be easily proven that the range of parameters $\omega, \varsigma$ identifying the full set of Bravais lattices is limited to the points displayed in gray in Figure 5-a. We refer to this region as the reduced domain of parameters. In fact, due to the symmetry properties of these lattices, the points that are outside this reduced domain characterize lattices which can always be parametrized with points in the reduced domain.

Figure 5-b depicts the Voronoi cells of the Bravais lattices in several points on the reduced domain $(\omega, \varsigma)$. Note that Oblique Bravais lattices are only represented by points in the interior of the reduced domain, while Rectangular, Square and Hexagonal lattices are only represented by points on its boundary. 


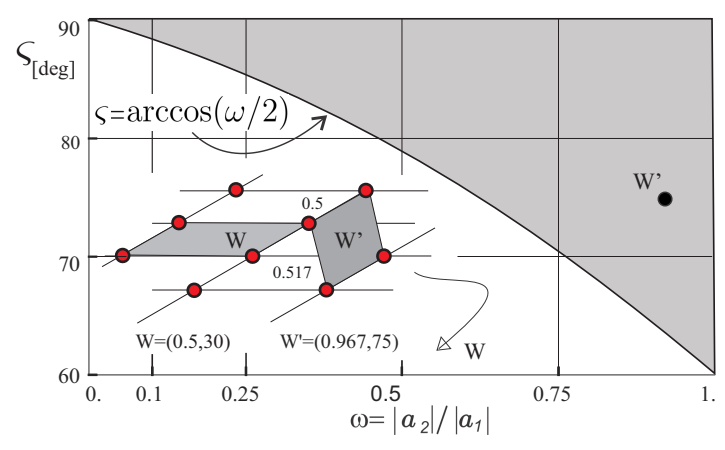

(a)

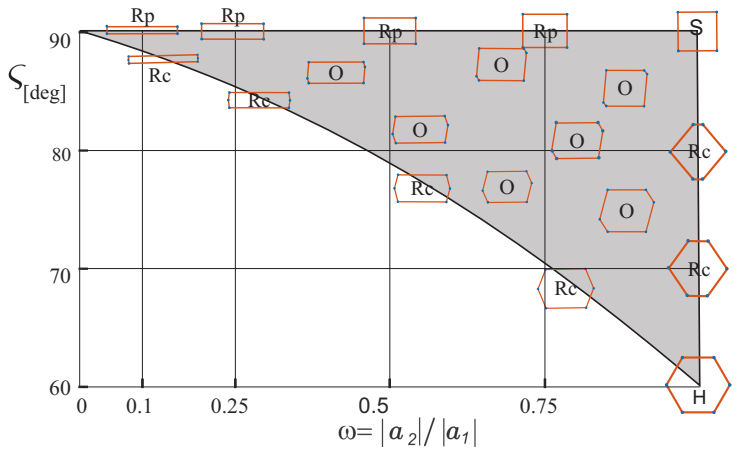

(b)

Figure 5: Space of parameters $(\omega, \varsigma)$ characterizing the Bravais lattices. a) The gray region is the reduced domain. Points outside of the reduced domain (W) identify lattices that can be parameterized with equivalent points in the reduced domain (W'). b) Voronoi cells of lattices characterized by different parameters $(\omega, \varsigma)$ : O (Oblique), Rc (Rectangular centered), Rp (Rectangular primitive), S (Square) and $\mathrm{H}$ (Hexagonal).

\subsubsection{Plane groups}

The material configuration, or crystal motif, can be defined by identifying a unit cell. When the motif is taken into account, an additional symmetry element, the glide reflection, has to be contemplated. It consists of a geometrical reflection, through a mirror line, followed by a translation, parallel to the same line. The translation distance is half of the periodicity distance, or unit cell size, parallel to the mirror line.

A plane group is the set of symmetry elements, including glide reflection, which identifies a wallpaper ${ }^{3}$. Here, we use the word wallpaper to denote a specific configuration of the material distribution of a periodic composite. Therefore, every wallpaper has an underlying Bravais Lattice and a motif that defines a plane group.

Similar to Bravais Lattice point groups, there are only a finite number of plane groups identifying all possible wallpapers. After introducing the motif and the glide reflection symmetries, the number of plane groups is seventeen. All of them are shown in Figure 6, where we depict the Voronoi cells of the underlying lattices with different motifs and the symmetry elements characterizing each plane group: reflection symmetry lines, the $n$-fold angle of rotational symmetry and the glide lines.

These plane groups are denoted by the letters $p$ or $c$ which indicates that the underlying lattice is either primitive or centered. The existence of one or two glide reflection lines are identified with the letters $g$ and $g g$, respectively. And, similar

\footnotetext{
${ }^{3}$ The words wallpaper and plane crystal have an identical meaning in this work.
} 
to the notation of point groups, the number identifies the n-fold angle of rotational symmetry, and the letters $m$ and $m m$ indicates if there are one or two mirror line systems, respectively.

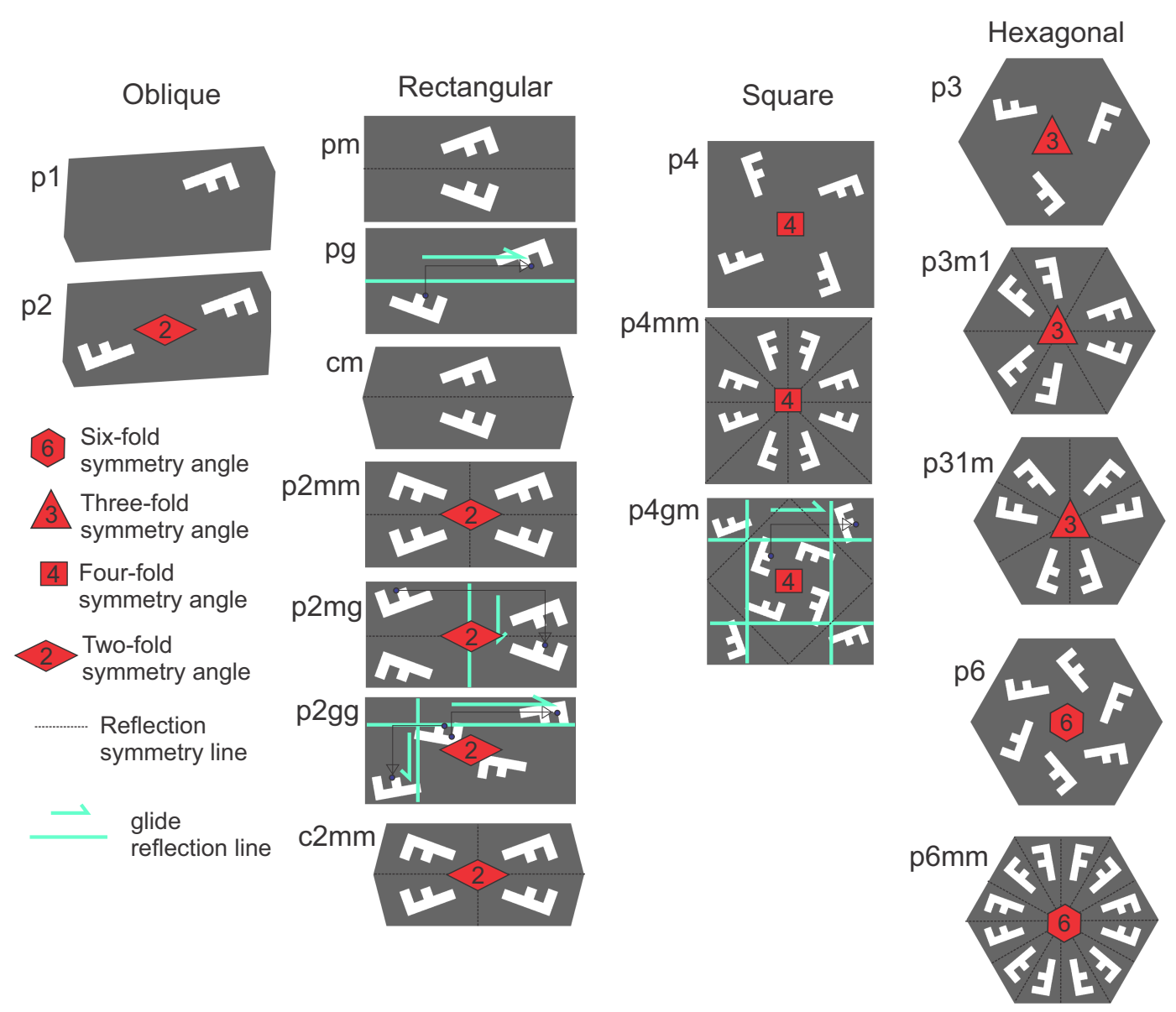

Figure 6: Voroni cells of crystals with the seventeen plane groups in 2D. The symmetry elements of each crystal, i.e. the Voronoi cell and the motif, are shown. Gray and white colors in the wallpapers represent the distribution of the composite phases.

The Bravais lattice symmetry of the wallpaper may be broken when the motif has a lower symmetry than that of the underlying lattice. Therefore, the wallpaper symmetry group is a subgroup of that characterizing the underlying lattice. According to this property, we next establish the relationship that assigns one point group to each one of the seventeen plane groups that represent arbitrary wallpapers and motifs. This relationship is shown in Table 2. Each plane group in the fourth column is associated (in the same line) with one point group in the third column. Note that wallpapers with plane group $p g$ has a point group $m$. This relationship comes from the fact that performing a mirror symmetry of a crystal with symmetry $p g$ results in the same crystal with a translation of half of the unit cell size parallel 
to the glide line. Therefore, by considering an infinite crystal, both crystals, the original one and the reflected and translated one, are indistinguishable when the effective material properties are evaluated. A similar conclusion can be given to wallpapers with plane group $p 2 m g, p 2 g g$ and $p 4 g m$ by changing the $g$ symmetry operation by a mirror symmetry operation $m$.

From these comments, there are only ten point groups characterizing the full set of wallpapers. They are depicted in Table 2, third column, and are : 1, 2, $m, 2 \mathrm{~mm}$, 4, $4 m m, 3,3 m m, 6$ and $6 m m$.

The system of crystals: the crystals are next classified by their point group symmetry. We identify all crystals which have a given point group. This identification is shown in Table 2, see also Landwehr [20]. Note that each crystal is composed of an underlying lattice, shown in column 5, and a motif having the symmetry given by the plane group in column 4. For example, crystals which lattices are Rectangular or Square and their plane groups are $p 2 m m, p 2 m g, p 2 g g$ and $c 2 m m$ have a point group $2 \mathrm{~mm}$. Also, observe that a crystal with hexagonal lattice and plane group $p 1$ has the point group 1 .

Once this relationship has been established, the systems of crystals can be defined as follows. A crystal system is the collection of crystals sharing the same point groups with an identical set of compatible lattices. Using this criterion, we can classify the crystals into four systems: Oblique, Rectangular, Square and Hexagonal ${ }^{4}$.

Again, as an example, the crystals having point groups $m$ and $2 m m$ are compatible with the same lattices, and therefore, they belong to the Rectangular crystal system.

\subsection{Elasticity tensor structures according with their sym- metries}

The symmetry of the overall elastic properties of heterogeneous materials are well established and are classified according to their point group, such as described in the work of Ting [21], see also [13].

The methodology followed by Ting to classify the elasticity tensor structures with comparable symmetries, a very conventional procedure in solid mechanics, consists of applying isometric transformations, compatible with a given point group, to the elasticity tensor. Then, the elastic tensor coefficients have to satisfy the necessary invariance conditions derived from these transformations.

In plane elasticity, this methodology determines four different elastic symmetry classes. They are denoted $O(2)$ for Isotropic, $D_{4}$ for Tetragonal, $D_{2}$ for Orthotropic

\footnotetext{
${ }^{4}$ In plane crystals, there is a one-to-one relationship between the lattice systems and the crystal systems. However, when the same classification is extended to three-dimensions, this one-to-one relationship is not preserved.
} 


\begin{tabular}{|c|c|c|}
\hline \multicolumn{10}{|c|}{ Elasticity tensors $\boldsymbol{C}_{N}$} \\
\hline $\left.\begin{array}{ccc}D_{4} & (4) \\
c_{1} & c_{2} & 0 \\
c_{2} & c_{1} & 0 \\
0 & 0 & \left(c_{1}-c_{2}\right)\end{array}\right] \quad\left[\begin{array}{ccc}c_{1} & c_{2} & 0 \\
c_{2} & c_{1} & 0 \\
0 & 0 & c_{3}\end{array}\right]$ & $D_{2}(5)$ \\
0 & {$\left[\begin{array}{ccc}c_{1} & c_{2} & 0 \\
c_{2} & c_{4} & 0 \\
0 & 0 & c_{3}\end{array}\right] \quad\left[\begin{array}{ccc}c_{1} & c_{2} & c_{5} \\
c_{2} & c_{4} & -c_{5} \\
c_{5} & -c_{5} & c_{3}\end{array}\right]$} \\
\hline
\end{tabular}

Table 1: a) Symmetry classes in plane elasticity. Structure of the elasticity tensors, $\boldsymbol{C}_{N}$, expressed in the Normal coordinates (material axis): $O(2)$ for Isotropic, $D_{4}$ for Tetragonal, $D_{2}$ for Orthotropic and $Z_{2}$ for fully Anisotropic materials. The total numbers of elastic coefficients $c_{1}, c_{2}, \ldots$ defining the elasticity tensors are shown in parenthesis for each symmetry class. The rotation angle transforming $\boldsymbol{C}$ into $\boldsymbol{C}_{N}$ is also considered as an additional coefficient of the elasticity tensor.

and $Z_{2}$ for fully Anisotropic materials. From higher to lower symmetry classes, they are: $O(2) \subset D_{4} \subset D_{2} \subset Z_{2}$. Furthermore, the point group with lower symmetry that is compatible with each one of these classes is: 3 for $O(2), 4$ for $D_{4}, m$ for $D_{2}$, and 1 for $Z_{2}$, respectively. This association between the four elastic classes and the point groups is shown in Table 2 ([21]).

The criterion followed to find the four classes of elastic symmetries implicitly introduces a coordinate system which is aligned with the symmetry planes of the point group. This coordinate system is called here as the Normal coordinate system. We use the notation $\boldsymbol{C}_{N}$ to indicate that the elasticity tensor is described in this coordinate system.

The Table 1 displays the elasticity tensor coefficients of $\boldsymbol{C}_{N}$ for the four elastic symmetry classes ${ }^{5}$. The numbers in parenthesis indicate the quantity of independent elastic coefficients that define the elasticity tensors.

Considering an arbitrary elasticity tensor $\boldsymbol{C}$ described in the Cartesian coordinate system, Auffray et al. [22] describe an algorithm to determine its elastic symmetry class as well as to computes the rotation angle transforming $\boldsymbol{C}$ into its normal form $\boldsymbol{C}_{N}$. See also the Appendix in the paper by Podestá et al. [23].

\subsection{Neumann's principle}

The connection existing between the symmetry elements of the micro-architecture geometry and the symmetry elements of the overall elasticity tensor can be stated with a fundamental postulate of the crystal physics known as Neumann's principle.

\footnotetext{
${ }^{5}$ Here, the elasticity tensor $\boldsymbol{C}_{N}$ is described as a matrix in Kelvin's notation. The stress and strain vectors in Kelvin's notations are given by: $\left[\sigma_{s s} ; \sigma_{n n} ; \sqrt{2} \sigma_{s n}\right]^{T}$ and $\left[\varepsilon_{s s} ; \varepsilon_{n n} ; \sqrt{2} \varepsilon_{s n}\right]^{T}$, respectively, where $s$ and $n$ are the Natural coordinates. Therefore, the components of the matrix $\boldsymbol{C}_{N}$ are the corresponding elastic coefficients that are defined in accordance with the notation of these vectors.
} 


\begin{tabular}{|c|c|c|c|c|c|c|c|c|}
\hline \multirow{2}{*}{$\begin{array}{c}\text { Elastic } \\
\text { symmetry } \\
\text { class }\end{array}$} & \multirow{2}{*}{$\begin{array}{l}\text { Crystal } \\
\text { system }\end{array}$} & \multirow{2}{*}{$\begin{array}{l}\text { Point } \\
\text { group }\end{array}$} & \multirow{2}{*}{$\begin{array}{l}\text { Plane } \\
\text { group }\end{array}$} & \multicolumn{5}{|c|}{ Compatible Bravais lattice and Wigner-Seitz unit cell } \\
\hline & & & & Oblique & $\begin{array}{l}\text { Rectangular } \\
\text { primitive }\end{array}$ & $\begin{array}{c}\text { Rectangular } \\
\text { centered }\end{array}$ & Square & Hexa \\
\hline \multirow{2}{*}{$\mathrm{Z} 2$} & \multirow{2}{*}{ oblique } & 1 & $\mathrm{p} 1$ & & & & & \\
\hline & & 2 & $\mathrm{p} 2$ & & & & & \\
\hline \multirow{7}{*}{ D2 } & \multirow{7}{*}{ rectangular } & \multirow{3}{*}{$\mathrm{m}$} & $\mathrm{pm}$ & & & & & \\
\hline & & & pg & & & & & \\
\hline & & & $\mathrm{cm}$ & & & & & \\
\hline & & \multirow{4}{*}{$2 \mathrm{~mm}$} & $\mathrm{p} 2 \mathrm{~mm}$ & & & & & \\
\hline & & & $\mathrm{p} 2 \mathrm{mg}$ & & & & & \\
\hline & & & p2gg & & & & & \\
\hline & & & $\mathrm{c} 2 \mathrm{~mm}$ & & & & & \\
\hline \multirow{3}{*}{$\mathrm{D} 4$} & \multirow{3}{*}{ square } & 4 & $\mathrm{p} 4$ & & & & & \\
\hline & & \multirow{2}{*}{$4 \mathrm{~mm}$} & $\mathrm{p} 4 \mathrm{~mm}$ & & & & & \\
\hline & & & $\mathrm{p} 4 \mathrm{gm}$ & & & & & \\
\hline \multirow{5}{*}{$\mathrm{O}(2)$} & \multirow{5}{*}{ hexagonal } & 3 & p3 & & & & & \\
\hline & & \multirow{2}{*}{$3 \mathrm{~m}$} & p3m1 & & & & & \\
\hline & & & $\mathrm{p} 31 \mathrm{~m}$ & & & & & \\
\hline & & 6 & p6 & & & & & \\
\hline & & $6 \mathrm{~mm}$ & $\mathrm{p} 6 \mathrm{~mm}$ & & & & & \\
\hline
\end{tabular}

Table 2: Elastic symmetry classes (column 1) are determined by the point group (column 3) of the crystal system (column 2). Also, each one of the seventeen plane groups (column 4), depicted in Tables 3 and 4, corresponds to a point group. Additionally, each plane group is compatible with several Bravais lattices (column 5). The symmetries (number of symmetry elements) increase from top to bottom. The higher symmetry is in the bottom of the Table. 
This principle states that "the symmetry elements of any effective physical property (optical, magnetic, thermal, mechanical properties) of a crystal must include the symmetry elements of the point group of the crystal", see Nye [13].

According to this principle, the relation between the point group and the elastic symmetry class is recognized in Table 2 between the first and third column. Note that, in the elastic case we are considering, identical lines of the Table relates an elastic class (column 1) with a crystal system (column 2).

This principle is confirmed by the computed results presented in Tables 3 and 4. In these Tables, the effective elasticity tensors of seventeen composites depicted in column 1 are shown. The micro-architectures of these composites show different plane groups. The Voronoi cells of these micro-architectures are shown in Figure 6 and their plane groups are displayed in column 2 of the Tables 3 and 4 . The related point group to each plane group is presented in column 3 of the same Tables.

The computed effective elasticity tensors in Normal coordinates ${ }^{6}$ are denoted by $\boldsymbol{C}_{N}^{h}$ and are presented in Column 5 of Tables 3 and 4 . In the same column, the angle between Cartesian and Normal coordinates are only shown for those cases where both coordinate systems are not coincident. According to the expressions of $\boldsymbol{C}_{N}^{h}$ in the Tables, and comparing with Table 1, we can identify their elastic symmetry class which are depicted in column 4. Also, and according to the same characterization of the elastic symmetry, the point groups of the effective elasticity tensors, $\boldsymbol{C}_{N}^{h}$, are compatible with the symmetry anticipated by Neumann's principle and the information given in Table 2. Finally, it is noted that the Normal axes coincide with the Cartesian ones when one of the symmetry axes of the plane group is parallel to one Cartesian axis.

Remark: Neumann's principle does not state that the symmetry elements of a physical property must be the same as the corresponding ones to the crystal point group. Very often, the physical properties may possess higher symmetry than the crystal point group. For example, some periodic materials with square unit-cells and motifs having a plane group $p 1$, with a point group 1, may have an isotropic effective elasticity. Examples of this feature are the micro-structures displayed in Figure 1 of reference [24] having a plane group $p 2 m g$, with a point group $2 m m$, and an isotropic effective elasticity tensor. See also Figure 11 below, case $S_{p m}$, instance 2 .

As a remarkable consequence of this principle, a procedure based on the following premise is developed: given the number of parameters characterizing the elastic response, or similarly, the symmetry class of the target elasticity tensor, we design

\footnotetext{
${ }^{6}$ The composites are constituted by a stiff material, displayed in gray color, and void displayed in white color. The elasticity tensor is normalized with an adequate Young's modulus of the stiff material to attain a coefficient $C_{11}$ with value 1 in all cases. The Poisson's ratio of the stiff material is 0.3 .
} 
the micro-structure by appealing to Voronoi cells and plane groups guaranteeing the attainment of effective properties having the same or higher symmetries to those of the target tensor.

\section{Database of elasticity tensors}

An additional ingredient introduced in the present inverse design methodology is the use of a database containing homogenized elasticity tensors. This database is built off-line by sampling a spectrum of periodic composite materials.

The homogenized elasticity tensors stored in the database are computed from composites with a stiff phase and void. Their micro-structures are identified with the Bravais lattices parameters $(\omega, \varsigma)$ which are defined by expressions $(4)$ and $(5)$. The motifs are determined by a set of hard material bars. These bars are distributed into the Voronoi cell of the lattice by following two patterns $\mathrm{P}$, denoted $P_{A}$ and $P_{B}$.

The pattern $P_{A}$ consists of a set of bars placed on the boundaries of the cell such as shown in Figure 7-a. Pattern $P_{B}$ is similar to $P_{A}$ but with a re-entrant architecture of bars in the vertices of the Voronoi cells, such as shown in Figure 7-b. The geometry of pattern $P_{B}$ is defined with the relative position of points $V 1, V 2$ and the size $r$ respect to the cell size. Patterns $P_{A}$ and $P_{B}$ are arbitrarily chosen. However, pattern $P_{A}$ is a simple topology with a lattice-type micro-architecture. This type of micro-architecture has attracted enormous interest in last years, see for example [25] and [26]. Also, the pattern $P_{B}$ is a lattice-type micro-architecture that is introduced to obtain elastic properties with negative Poisson's ratios ([27], [28]). The bar thickness $e$ in both patterns $P_{A}$ and $P_{B}$ defines the volume fraction $f$ of the composite.

In this way, the database contains homogenized elasticity tensors $\boldsymbol{C}_{d b}^{h}$ of materials whose micro-structures are defined by four parameters $\omega, \varsigma, P$ and $f$. Therefore, each element of the database is identified through its dependence with these parameters, $\boldsymbol{C}_{d b}^{h}(\omega, \varsigma, P, f)$. The range of parameters $(\omega, \varsigma, P, f)$ that is utilized to compute the database are the following:

- The first two parameters, $\omega$ and $\varsigma$, are varied such that the parametrized reduced domain of Figure 5-a is swept by defining a regular mesh of 6283 sampling points which cover the full reduced space.

- Several volume fractions $f$ varying from $f=0.005$ to $f=0.8$. The volume fraction is given in term of the thickness $e$ of the bars.

- The variable $\mathrm{P}$ takes the values, $P_{A}$ and $P_{B}$.

The stiff material properties utilized to build the database are defined with a normalized Young's modulus $E=1 . G P a$ and Poisson's ratio $\nu=0.3$. 


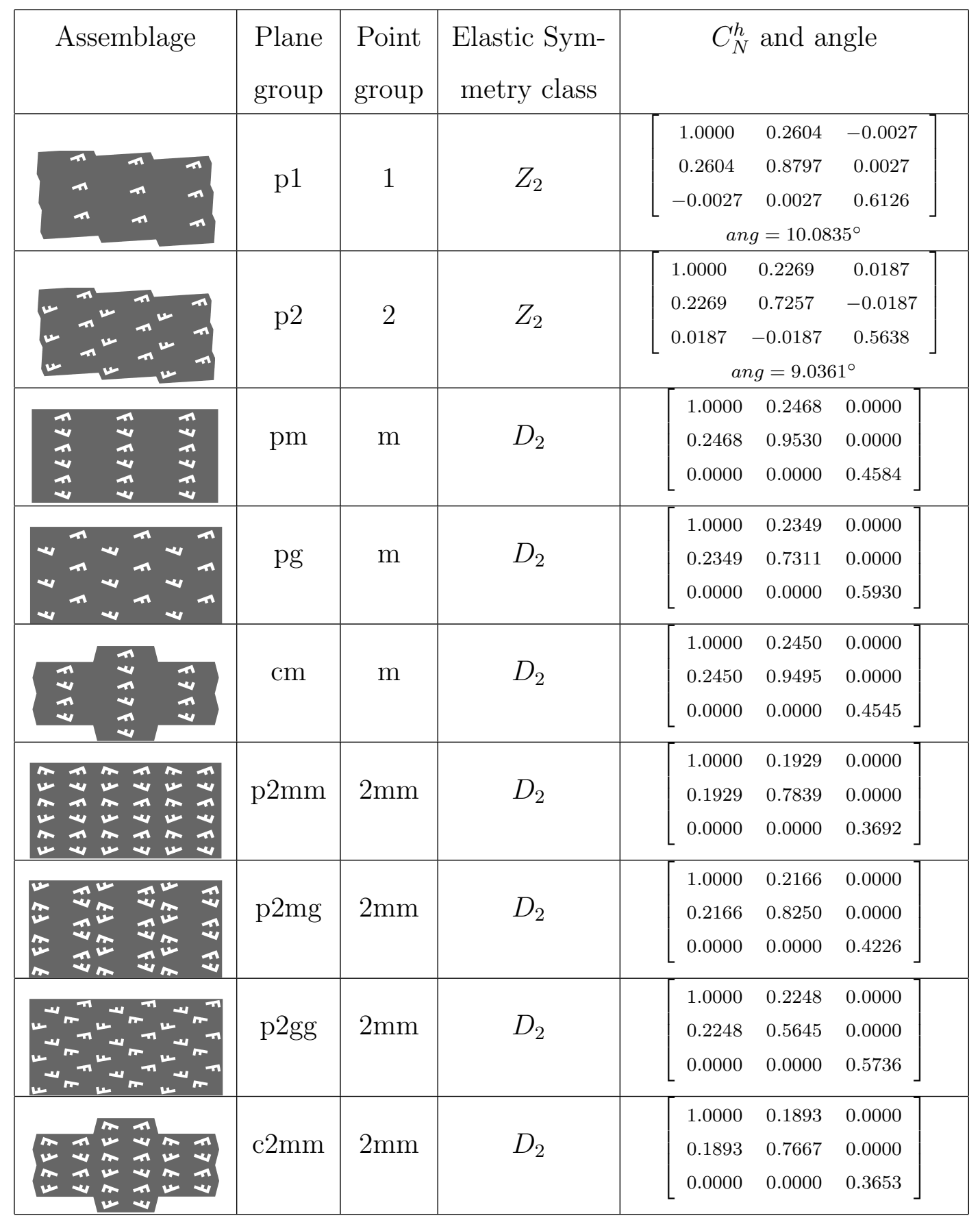

Table 3: Column 1 depicts the microstructure of the composites resulting from the assemblage of the Voronoi cells in Figure 6. These assemblages show the seventeen types of plane groups. Columns 2 and 3 depict the point and plane groups, respectively, of the assemblages. The column 5 presents the homogenized elasticity tensors in Natural coordinates, $\boldsymbol{C}_{N}^{h}$, and the rotation angle from Cartesian to Natural coordinates, of the composites in column 1. These homogenized tensors have the symmetry classes depicted in column 4. 


\begin{tabular}{|c|c|c|c|c|c|c|}
\hline Assemblage & $\begin{array}{l}\text { Plane } \\
\text { group }\end{array}$ & $\begin{array}{l}\text { Point } \\
\text { group }\end{array}$ & $\begin{array}{l}\text { Elastic Sym- } \\
\text { metry class }\end{array}$ & \multicolumn{3}{|c|}{$\begin{array}{c}C_{N}^{h} \\
\text { and angle }\end{array}$} \\
\hline $\begin{array}{ll}5 \\
5\end{array}$ & $\mathrm{p} 4$ & 4 & $D_{4}$ & $\begin{array}{c}1.0000 \\
0.2378 \\
0.0000 \\
\text { ang }=\end{array}$ & $\begin{array}{c}0.2378 \\
1.0000 \\
0.0000 \\
=-80.0\end{array}$ & $\begin{array}{c}0.0000 \\
0.0000 \\
0.6594 \\
70^{\circ}\end{array}$ \\
\hline & $\mathrm{p} 4 \mathrm{~mm}$ & $4 \mathrm{~mm}$ & $D_{4}$ & $\begin{array}{l}1.0000 \\
0.2803 \\
0.0000\end{array}$ & $\begin{array}{l}0.2803 \\
1.0000 \\
0.0000\end{array}$ & $\begin{array}{l}0.0000 \\
0.0000 \\
0.6515\end{array}$ \\
\hline & $\mathrm{p} 4 \mathrm{gm}$ & $4 \mathrm{~mm}$ & $D_{4}$ & $\begin{array}{l}1.0000 \\
0.2444 \\
0.0000\end{array}$ & $\begin{array}{l}0.2444 \\
1.0000 \\
0.0000\end{array}$ & $\begin{array}{l}0.0000 \\
0.0000 \\
0.6972\end{array}$ \\
\hline & p3 & 3 & $O(2)$ & $\begin{array}{l}1.0000 \\
0.2784 \\
0.0000\end{array}$ & $\begin{array}{l}0.2784 \\
1.0000 \\
0.0000\end{array}$ & $\begin{array}{l}0.0000 \\
0.0000 \\
0.7216\end{array}$ \\
\hline & p3m1 & $3 \mathrm{~m}$ & $O(2)$ & $\begin{array}{l}1.0000 \\
0.2991 \\
0.0000\end{array}$ & $\begin{array}{l}0.2991 \\
1.0000 \\
0.0000\end{array}$ & $\begin{array}{l}0.0000 \\
0.0000 \\
0.7009\end{array}$ \\
\hline & p31m & $3 \mathrm{~m}$ & $O(2)$ & $\begin{array}{l}1.0000 \\
0.3016 \\
0.0000\end{array}$ & $\begin{array}{l}0.3016 \\
1.0000 \\
0.0000\end{array}$ & $\begin{array}{l}0.0000 \\
0.0000 \\
0.6984\end{array}$ \\
\hline & p6 & 6 & $O(2)$ & $\begin{array}{l}1.0000 \\
0.2068 \\
0.0000\end{array}$ & $\begin{array}{l}0.2068 \\
1.0000 \\
0.0000\end{array}$ & $\begin{array}{l}0.0000 \\
0.0000 \\
0.7932\end{array}$ \\
\hline 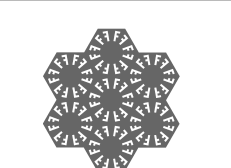 & p6mm & $6 \mathrm{~mm}$ & $O(2)$ & $\begin{array}{l}1.0000 \\
0.3401 \\
0.0000\end{array}$ & $\begin{array}{l}0.3401 \\
1.0000 \\
0.0000\end{array}$ & $\begin{array}{l}0.0000 \\
0.0000 \\
0.6599\end{array}$ \\
\hline
\end{tabular}

Table 4: (Continuation of Table 3). 


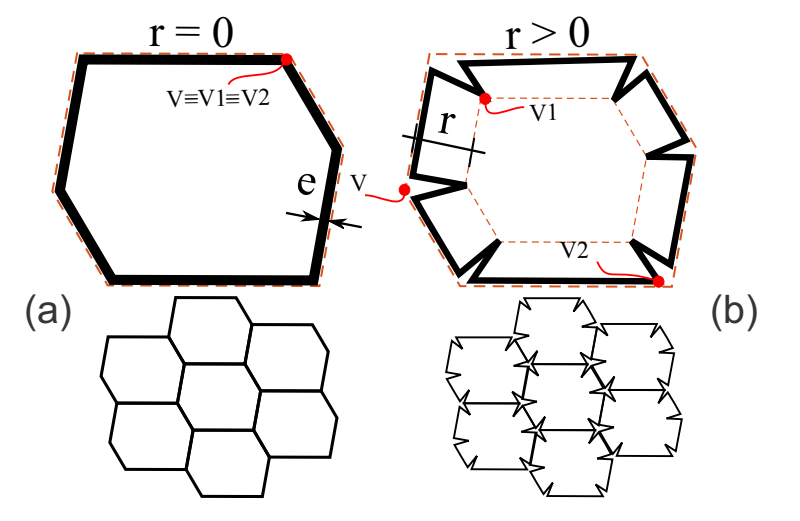

Figure 7: Two-parametrized topologies used for the database construction. Patterns: a) $P_{A}$, and b) $P_{B}$.

Partial results of this database have been presented in [23]. From that work, it can be observed that auxetic materials are captured with the micro-architectures characterized by the pattern $P_{B}$.

Remark: The information stored in the database, of the order of $10^{5}$ elasticity tensors, is utilized to provide a hint for selecting, from a predefined Bravais lattice system, the Voronoi cell used as the design domain of the topology optimization problem, see item (3) of the next Section 4. The database information is managed as follows:

- Once the target elasticity tensor $\hat{\boldsymbol{C}}_{N}$ and its point group is known, we search the parameters, $\zeta$, of the database entry whose elasticity tensor $\boldsymbol{C}_{d b}^{h}(\zeta)$ satisfies the solution of the problem:

$$
\zeta=\arg \left\{\min _{\zeta}\left\|\boldsymbol{C}_{d b}^{h}(\zeta)-\frac{1}{E_{1}} \hat{\boldsymbol{C}}_{N}\right\|\right\} ; \quad \text { where } \zeta:=\{\omega, \varsigma, P, f\},
$$

with the parameters $(\omega, \varsigma)$ restricted to the region characterizing Bravais lattices with identical point group to that of the target tensor. Regions in the plane $(\omega, \varsigma)$ with the related point groups are shown in Figure 5-b. The factor $E_{1}$ scaling the target elasticity tensor is the Young's modulus of the composite stiff phase to be designed.

- Furthermore, the micro-architecture associated with the solution of problem (6) could be taken as the initial configuration for the iterative topology optimization algorithm.

The database of homogenized elasticity tensors, the software to compute the entries of the database for the parameter set $\zeta$ and the software to attain the solution of 
the problem (6) are freely available in the Mendeley dataset repository, see [29]. All the codes are written in Matlab.

\section{A systematic procedure for tailoring elastic com- posites through inverse homogenization tech- nique}

Based on the symmetry properties of the target elasticity tensors, the discussion given in Section 2 and taking into account the elasticity tensor database, four heuristic rules are introduced to facilitate and enlarge the possible set of configurations obtained with the inverse homogenization design problem. These rules define a systematic procedure that can be adopted by the designer and are established as follows.

1) The topology optimization problem (2) is solved in the Normal basis of the target tensor. It means that the tensor $\hat{\boldsymbol{C}}$ is replaced by $\hat{\boldsymbol{C}}_{N}$ in the formulation (2).

2) Voronoi cells of Bravais lattices, whose point groups are compatible with the point groups of the target tensors, are chosen as the design domain $\Omega_{\mu}$. The relationship between the point groups of both entities, lattice and elasticity tensor, are shown in Table 2. Entities sharing the same point group in the Table are displayed in identical lines. For example, for a target tensor having $D_{2}$ symmetry, the compatible cells are those associated with $R_{p}$ or $R_{c}$ Bravais lattices, such as rectangles or irregular hexagons are shown in Figure 4 . In this case, note that square and regular hexagonal cells are also compatible.

3) The slenderness of the cell, i.e., the aspect ratio between the larger and shorter size of the cell, can be determined through the database entries and the criterion defined by expression (6). Typically, this slenderness ratio should be defined in the Voronoi cells of Oblique, $R_{c}$ and $R_{p}$ lattices.

4) The symmetry displaying the material distribution within the Voronoi cell is decided in accordance with symmetry properties of the target elasticity tensor. The material configuration symmetry defines the micro-architecture plane group. Then, this configuration should be compatible with the target elasticity tensor point group. The compatibility relationships between plane groups and elasticity tensor point groups are presented in Table 2; the entries displayed in the first and the fourth columns of the same line are compatible. Thus, recalling the previous example, i.e., a target tensor having $D_{2}$ symmetry, the compatible plane groups are $p m, p g, \mathrm{~cm}, p 2 m m, p 2 m g, p 2 g g$ or $c 2 m m$. 
Our experience is that several configurations, with compatible plane groups, have to be tested and solved. In some cases, improved designs are attained by enforcing the higher compatible symmetry, though, this is not a general rule.

To apply the last rule, the symmetries of the material configuration compatible with the plane group can only be enforced if the topology optimization problem is solved in the Normal basis of $\hat{\boldsymbol{C}}$, such as mentioned in the first rule. In this basis, the symmetry planes of the plane groups are coincident with the symmetry axes of the Voronoi cells, such as shown in Figure 6. Note also that the cost of the finite element analysis for computing the homogenized elasticity tensor, in the topology optimization problem, can significantly be reduced after introducing the symmetries compatible with the plane group. This issue has been addressed by Barbarosie et al. [11] and Lukkassen et al. [12].

\section{$5 \quad$ Numerical assessments}

Some micro-structures designed via a topology optimization problem that follows the systematic procedure previously described are analyzed and compared with microstructures obtained with alternative conventional procedures without enforcing those rules. The effects that several design variables have on the attained solutions are assessed, such as:

i) Different types of design domains. The tested cell shapes are square, rectangles with different aspect ratios and regular or irregular hexagons. Additionally, the enforcement of different plane groups is also tested.

ii) Voronoi cells with symmetry axes arbitrarily placed or aligned with the Normal bases are tested.

iii) Different material configurations are taken as the starting points of the topology optimization algorithm.

The objective is to compare the solutions obtained in those different situations.

\subsection{Designing an extreme isotropic material with minimum Poisson's ratio}

The target material, whose micro-structure is designed in this test, is a biphasic isotropic composite with the minimum Poisson's ratio that is estimated using the Cherkaev and Gibiansky [30] bounds for this kind of composite. The properties of the composite components and the volume fraction of the hard material are chosen 
such that the Cherkaev et al.'s bounds estimate an elasticity tensor with negative Poisson's ratio, which makes this test more challenging.

Due to the interesting properties of auxetic materials, their micro-architecture designs have been profusely studied in the literature, such as mentioned in the review by Ren et al. [31]. Only to cite a few works closely related to the present contribution, the paper of Larsen et al. [32] describes the micro-architecture design of auxetic composites using topology optimization tools, particularly the SIMP technique. This paper also shows the manufacture of such composites and their experimental testing. More recently, and using a similar development, Andreassen et al. [24] have reported the designs of 3D micro-architectures with negative Poisson's ratios and describe their manufacture attainability. Also, the work by Jiang and Li [28] gives a survey of this topic, as well as a discussion about the fabrication of auxetic materials. An interesting analysis of three typical micro-architecture topologies, re-entrant, chiral and rotating, that can be used for designing auxetic materials has been reported in the work by Kolken and Zadpoor [27], while a family of lattice-like metamaterials, with macroscopic effective Poisson's ratio arbitrarily close to the stability limit -1 , has been studied by Sigmund [2] and more recently by Cabras and Brun [33]. Finally, the initial contributions on auxetic materials of Lakes and Evans and co-workers in the 80's and 90's must be here mentioned, see for example Evans and Alderson [34] and reference cited therein.

\subsubsection{Studied case}

An isotropic composite constituted by two phases $M_{1}$ and $M_{2}$ is assumed. Material $M_{1}$ has a bulk modulus ${ }^{7} K_{1}=5 / 7[G P a]$ and shear modulus $G_{1}=5 / 13[G P a]$. Material $M_{2}$ has a bulk modulus $K_{2}=K_{1} / 200[G P a]$ and shear modulus $G_{2}=$ $G_{1} / 200[G P a]$. The volume fraction of $M_{1}$ is $f_{1}=0.5$. Plane strain hypothesis is considered.

According to the Cherkaev et al.'s analysis, the effective moduli of an isotropic composite constituted by $M_{1}$ and $M_{2}$ is bounded below and above with the curves plotted in Figure 8-a, where $\hat{K}$ and $\hat{G}$ are the effective bulk and shear moduli of the composite, respectively. Taking into account these bounds, the minimum possible Poisson's ratio for such composites ${ }^{8}$ is attained at the point satisfying:

$$
\min \frac{\hat{K}-\hat{G}}{\hat{K}+\hat{G}},
$$

with $\hat{K}$ and $\hat{G}$ bounded by the domain specified by the Cherkaev et al.'s analysis. This point should be on the curve $A B C$. The Poisson's ratios of points

\footnotetext{
${ }^{7}$ The plane strain bulk modulus $K$ is given by $K=\kappa+G / 3$, where $\kappa$ and $G$ are the conventional bulk and shear moduli of the three-dimensional theory.

${ }^{8}$ The effective Poisson's ratio of plane elasticity theory if given by: $\hat{\nu}^{*}=(\hat{K}-\hat{G}) /(\hat{K}+\hat{G})$.
} 


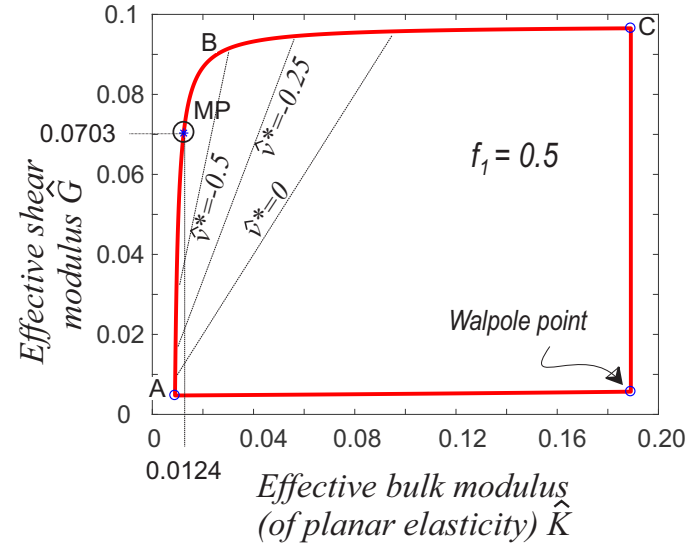

(a)

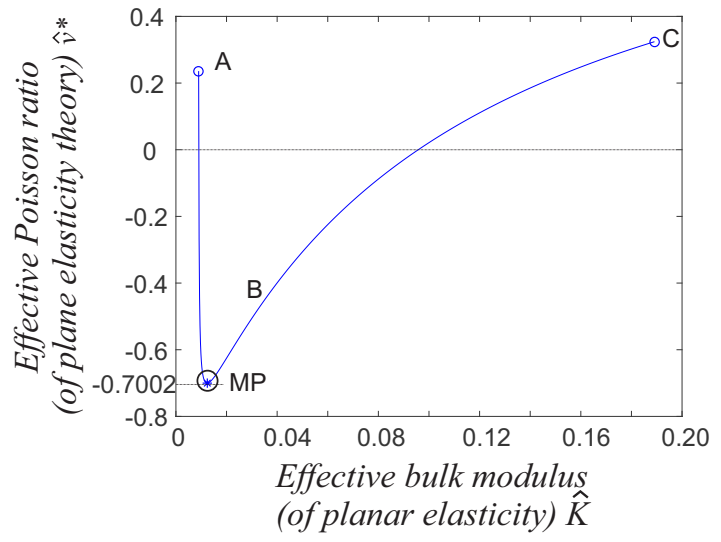

(b)

Figure 8: a) Bounds of effective properties for a two-phase isotropic composite according to Cherkaev et al. Properties of phases $M_{1}$ and $M_{2}$ are $K_{1}=5 / 7 ; K_{2}=$ $K_{1} / 200 ; G_{1}=5 / 13 ; G_{2}=G_{1} / 200$ (units of moduli in GPa). Volume fraction of phase $M_{1}$ is $f_{1}=0.5$. Iso-lines of $\hat{\nu}^{*}$ with values $0,-0.25$ and -0.5 are shown. b) Poisson's ratios of isotropic composites whose effective properties are characterized by the points on the curve $\mathrm{ABC}$ in a).

on that curve are plot in Figure 8-b. The point MP with coordinates $(\hat{K}, \hat{G})=$ $(0.012404,0.070334)$ is the one satisfying (7) with the minimum Poisson's ratio $\hat{\nu}^{*}=-0.7002$. Then, the effective elasticity tensor that is related to the MP point results:

$$
\hat{\boldsymbol{C}}_{N}=\left[\begin{array}{ccc}
0.082739 & -0.057930 & 0 \\
-0.057930 & 0.082739 & 0 \\
0 & 0 & 0.140669
\end{array}\right] \text {, }
$$

which is taken as the target elasticity tensor to formulate the topology optimization problem. Note that this tensor is isotropic, and therefore, it is given in the Normal coordinates.

\subsubsection{The topology optimization algorithm}

The volume fraction constraint $\left(f_{1}=0.5\right)$ is satisfied in the present case by redefining the objective function of the optimization problem (2) as follows

$$
\min _{\chi}\left(\frac{1}{\left|\Omega_{\mu}\right|} \int_{\Omega_{\mu}} \chi d \Omega-f_{1}\right)^{2} .
$$

Problem (2) with the above objective function is solved with a topology optimization technique that uses the concepts of topological derivative and level-set 
function, similar to the procedure explained in the Amstutz's works, see [35] and [5]. A brief summary of this technique is given in Appendix A. Also, considering that the target tensor $\hat{\boldsymbol{C}}_{N}$ may not be attainable, the convergence of the algorithm is reached when the following conditions are satisfied: i) the error in the normalized constraint $\left\|\boldsymbol{C}^{h}-\hat{\boldsymbol{C}}\right\| /\|\hat{\boldsymbol{C}}\|$ has stabilized to a value less than $\operatorname{tol}_{C}=0.4$, and ii) the criterion (25) of the Appendix A has stabilized to a value greater than $1-t_{o} l_{\psi}$ with ${ }^{t o l} l_{\psi}=0.1$. Stabilization in both values means that they do not substantially change during the last 10 iterations.

To facilitate the comparative analysis in these cases, the topology optimization algorithm forces solutions with material configurations displaying only one length scale. Therefore, the isotropic homogeneous phases are spatially distributed in such a way that their finest width is limited below by a size of the order of the micro-cell size. Under this condition, typical layered sub-micro-structures are topologies not admitted. This criterion is imposed on the topology optimization algorithm through a conventional Helmoltz's type spatial filter that is described by equation (26) in Appendix A, see additional details in [36] and [37] for local length scale control. The filter size $r_{l s}$ is constant and identical in all computed cases.

\subsubsection{Voronoi cells, symmetries and stiff phase configurations for initial- izing the topology optimization algorithm}

Considering that the target composite is isotropic, and following the procedure proposed in Section 4 and Table 2, the design domain of the topology optimization problem could be taken as a regular hexagon. Also, the material distribution could be compatible with the plane groups $p 3, p 3 m 1, p 31 m, p 6$ or $p 6 m m$. These choices guarantee the isotropy of the designed material. Particularly in this Section, we test the Hexagonal cell with $p 1, p 3$ and $p 6$ plane groups. We additionally test a square cell enforcing symmetries consistent with $p 1$ and $p m$ plane groups.

The five tested cases are identified as follows: $H_{p 1}, H_{p 3}$ and $H_{p 6}$ refer to hexagonal cells with plane groups $p 1, p 3$ and $p 6$, respectively. $S_{p 1}$ and $S_{p m}$ refer to square cells with plane groups $p 1$ and $p m$, respectively. In the cases with $p 1$ and $p m$ plane groups, the isotropy of the designed material is not guaranteed.

In order to attain a representative response of the computational procedure, ten instances of each one of these five tested cases are solved with the iterative topology optimization algorithm. So, they are a total of 50 designs. All of them are initialized

with random distributions of the stiff phase satisfying the condition $f_{1}=0.5$. Note that the item (3) of Section 4 is not brought into play for solving these cases. 

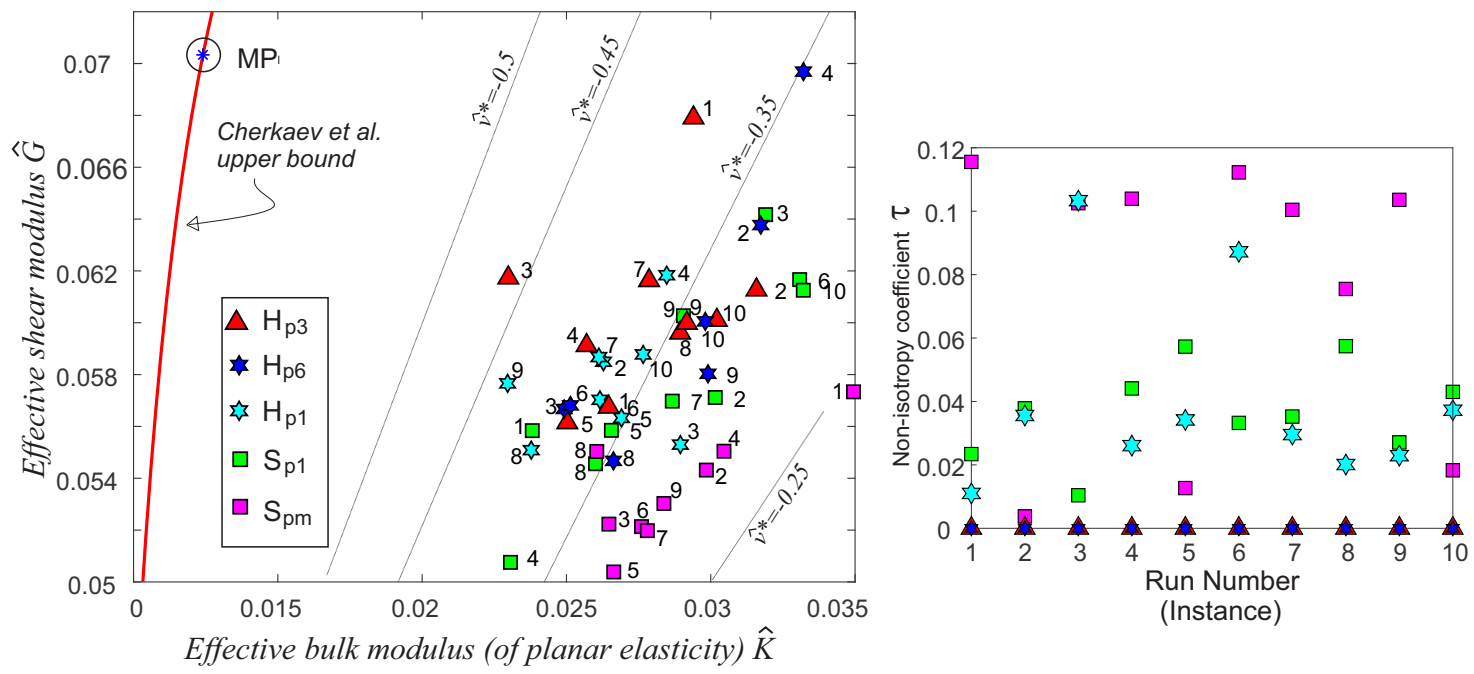

Figure 9: Micro-structure design of a composite with negative Poisson's ratio. Three design cases denoted $H_{p 1}, H_{p 3}$ and $H_{p 6}$ are solved with hexagonal cells and $p 1, p 3$ and $p 6$ plane groups. Two cases with square cells denoted $S_{p 1}$ and $S_{p m}$ are solved with $p 1$ and $p m$ plane groups. Ten instance of each one of the five cases have been solved. a) Solutions plotted in the space of the effective bulk and shear moduli. Diferent instances of each plane group are identified with a run number. The isolines of effective Poisson's ratios of plane elasticity theory $\hat{\nu}^{*}=-0.25,-0.35,-0.45$ and -0.5 are shown; b) non-isotropy coefficients that correspond to the complete set of solutions.

\subsubsection{Numerical results}

The effective moduli of the 50 solved micro-architectures are depicted in the space defined by the effective shear modulus and effective bulk modulus shown in Figure 9-a. The Cherkaev et al.'s bounds and the MP point are also depicted in the plot. In the cases $H_{p 1}, S_{p 1}$ and $S_{p m}$, where isotropy cannot be guaranteed, the parameters $\hat{K}$ and $\hat{G}$ are computed using the expression (11) below. In Figure 9, five different symbols identify the five cases, while the numbers (from 1 to 10 ) beside the symbols identify the corresponding instances of each case.

We remark that all of the 50 designs have been solved with the same filter to avoid micro-structure solutions displaying subscales.

The isotropy of $\boldsymbol{C}^{h}$ can be estimated through the non-isotropy coefficient $\tau$ that measures its distance to the space of isotropic tensors (see [8]):

$$
\tau=\frac{\left\|\boldsymbol{C}^{h}-\boldsymbol{C}^{i s o}\right\|}{\left\|\boldsymbol{C}^{h}\right\|}
$$

where $\boldsymbol{C}^{i s o}$ is considered as a projection of $\boldsymbol{C}^{h}$ onto the space of isotropic tensors 
and is computed with the formula:

$$
\boldsymbol{C}^{i s o}=\left[\begin{array}{ccc}
K^{i s o}+G^{i s o} & K^{i s o}-G^{i s o} & 0 \\
K^{i s o}-G^{i s o} & K^{i s o}+G^{i s o} & 0 \\
0 & 0 & 2 G^{i s o}
\end{array}\right]
$$

where

$$
\begin{aligned}
K^{i s o} & =\frac{1}{8}\left(3 C_{11}^{h}+3 C_{22}^{h}+2 C_{12}^{h}+2 C_{33}^{h}\right)-G^{i s o}, \\
G^{i s o} & =\frac{1}{8}\left(C_{11}^{h}+C_{22}^{h}-2 C_{12}^{h}+2 C_{33}^{h}\right) .
\end{aligned}
$$

Expressions (11) have been taken from Meille and Garboczi [38].

The so-defined non-isotropy coefficient $\tau$ has been computed for the complete set of solutions. Figure 9-b plots these coefficients. These results confirm that the solutions obtained with the $p 3$ and $p 6$ plane groups are exactly isotropic $(\tau=0)^{9}$.

Figure 10 presents the errors of the solutions to satisfy the constraint condition of the problem (2). Each sub-Figure display the normalized distance between the target elasticity tensor $\hat{\boldsymbol{C}}_{N}$ and the homogenized elasticity tensor $\boldsymbol{C}^{h}$ of each microarchitecture design case. Note that this distance is not necessarily equal to the distance between the point MP and each solution point displayed in Figure 9-a. For example, compare in both Figures the results of instances 2 and 8 with plane group $S_{p m}$. Figure 10-b displays that the error for instance 8 is lower than the error for instance 2. However, the contrary effect is observed in Figure 9-a. This result comes from the fact that Figure 10 disregards the isotropy property of the obtained homogenized elasticity tensors.

Figure 11 displays some designed micro-structures. The plot of Figure 9-a is repeated in Figure 11 but only including the instances whose micro-structure are shown in this Figure.

\subsubsection{Discussion of results}

From the analysis of the solutions obtained in these tests, it can be drawn some observations:

- Solutions obtained by enforcing the $H_{p 3}$ plane group are the closer ones to the MP-point. This conclusion is attained with the distances computed with the

\footnotetext{
${ }^{9}$ Computational homogenization of solutions obtained with $p 3$ and $p 6$ plane groups and using unit cell finite element meshes that preserve the symmetry $p 3$ or $p 6$ provides a value $\tau$ being exactly zero to the machine precision.
} 

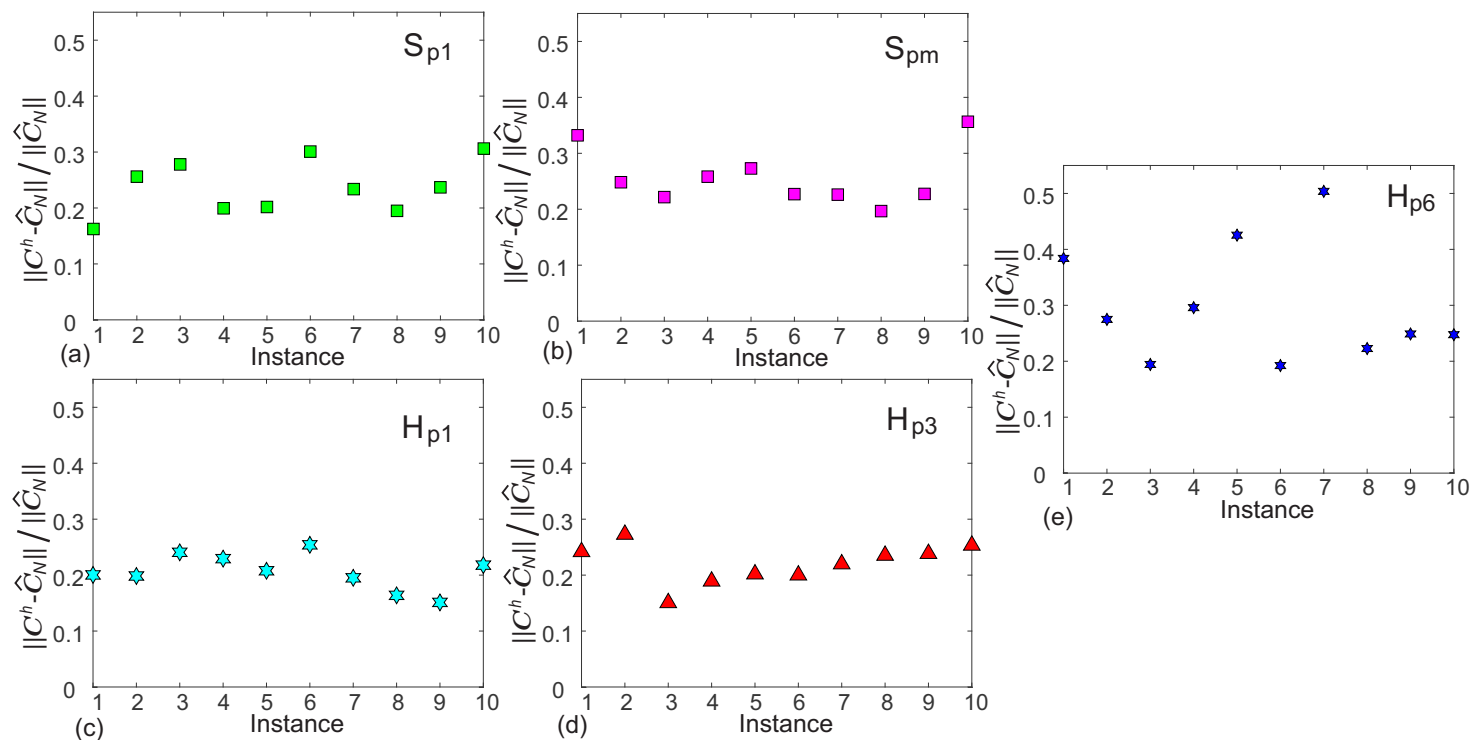

Figure 10: Normalized errors to satisfy the constraint $\left\|\boldsymbol{C}^{h}-\hat{\boldsymbol{C}}_{N}\right\|$. Plane groups: a) $S_{p 1}$; b) $S_{p m}$; c) $H_{p 1}$, d) $H_{p 3}$, e) $H_{p 6}$.

metric induced by (9)-(11). Solutions with $H_{p 3}$ plane group have intermediate material configuration symmetries, between $H_{p 1}$ and $H_{p 6}$ plane groups. Alternatively, the farther solutions with hexagons have been obtained by enforcing the higher symmetry, i.e., $H_{p 6}$ plane group. However, this result cannot be taken as a general conclusion. As a counterexample, we remind the case of the composite designed to attain the Walpole point, see Sigmund [10]. In this case, it has been shown that enforcing the $H_{p 6 m m}$ plane group, instead of $H_{p 3}$, provides better solutions, see Méndez et al. [39].

- The double bar mechanisms depicted in Figure 11, case $H_{p 3}$ instances 1 and 3 , may suggest for auxetic materials a similar observation to that reported by [10], in the sense that better structures are obtained by splitting individual bars into "multiply laminated bars". In fact, Sigmund proposed a very general class of optimal stiffness microstructures based on this principle which achieves the maximum energy bounds. Even when, we cannot guarantee this conclusion in the present study, this important aspect of auxetic material designs with isotropic properties deserves additional research by repeating the design process with finer grids to generate a sequence of solutions tending to the theoretical value.

- Chiral materials ([27],[28]) appear to be the micro-architecture topologies attained when enforcing the $H_{p 6}$ plane group. This type of architecture can be observed in instances 6 and, though less clearly, in instances 2 and 3. Also, 

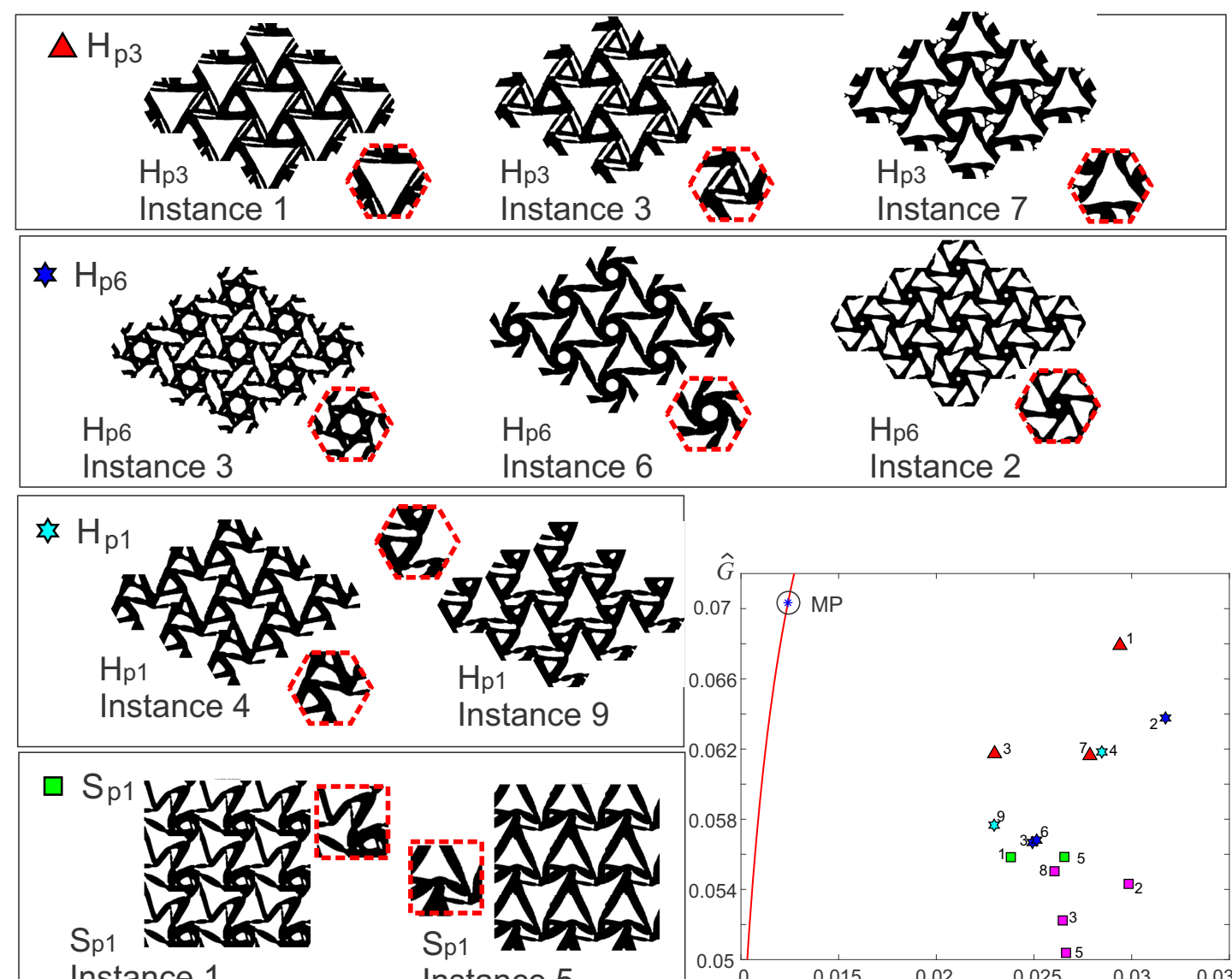

Instance 1
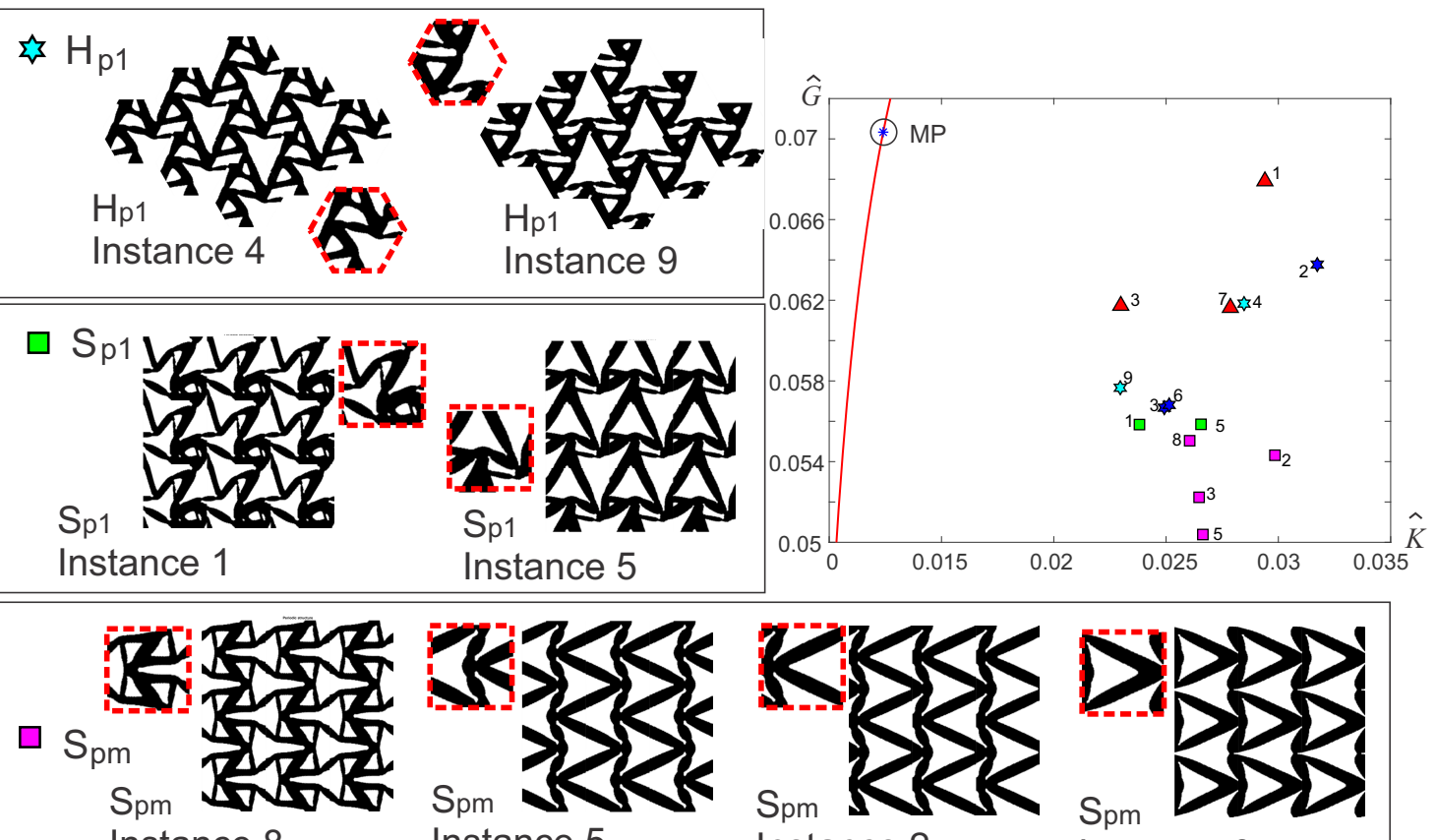

Instance 8
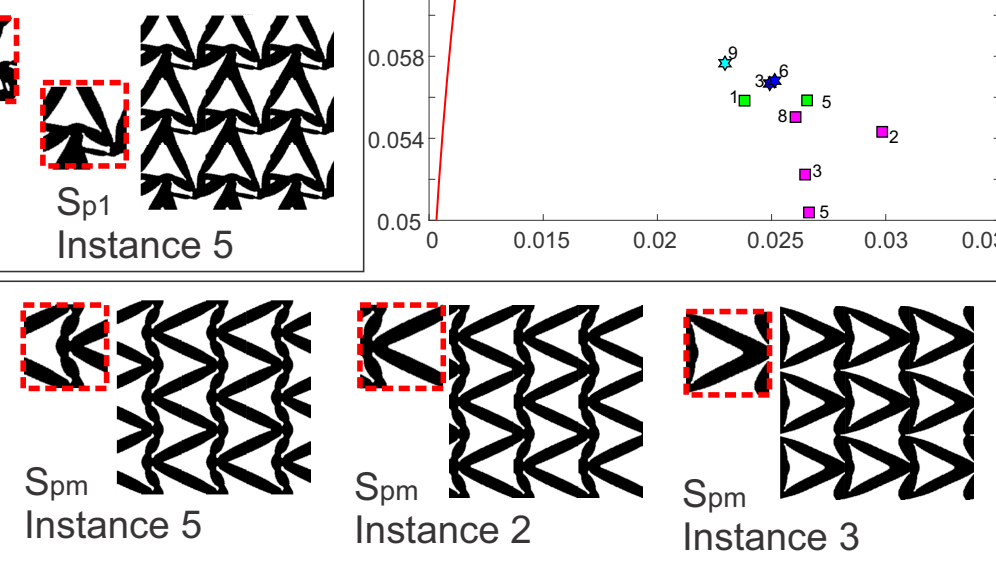

Figure 11: Several instances of the designed micro-structures of a composite with negative Poisson's ratio. plane groups $H_{p 1}, H_{p 3}, H_{p 6}, S_{p 1}$ and $S_{p m}$. Composites with phases $M_{1}$ and $M_{2}$ and properties given by: $K_{1}=5 / 7 ; K_{2}=K_{1} / 200 ; G_{1}=5 / 13$; $G_{2}=G_{1} / 200$ (units of moduli in GPa). The volume fraction of phase $M_{1}$ is $f_{1}=0.5$. 
there can be observed a tendency for capturing chirality in the solution $S_{p 1}$, instance 1.

- The attainment of isotropy with $H_{p 1}, S_{p 1}$ and $S_{p m}$ plane groups lies purely on the effectiveness of the topology optimization algorithm. Some solutions of $S_{p m}$ plane group show micro-structures whose effective responses are closely isotropic. However, this is not the general response for these plane groups.

- Solutions obtained with $S_{p m}$ plane group, instances 2 and 5, display a similar micro-architecture to the one previously reported in the literature, see Larsen et al. [32] and Andreassen et al. [24]. As can be observed in Figure 9-b and ignoring a small error, both particular instances (2 and 5) display isotropic effective elastic responses, agreeing, therefore, with the reported results in Andreassen et al. [24].

- Micro-architecture configurations with higher symmetries appear to be more easily parametrized.

- Solutions obtained by enforcing the $S_{p 1}$ plane group indicate a preference to attain topologies with higher symmetries, compatible with the $S_{p m}$ plane group. An identical conclusion can be drawn for the solutions obtained by enforcing the $H_{p 1}$ plane group; these topologies have a preference to attain symmetries compatible with the $H_{p 3}$ plane group. It is particularly observed that the solutions of instance 2,5 and 8 , of the case $S_{p m}$, present a vertical glide plane. Therefore, these micro-structures result with a $S_{p 2 m g}$ plane group which is a higher symmetry than the one originally imposed.

- It is also important to remark that the number of optimal topologies in the design problem decreases by enforcing higher symmetries. For example, noncentered or flipped topologies in the base cell can be avoided by enforcing that the symmetry lines cross the central point of the cell.

\subsection{Micro-structure designs of materials with $D_{2}$ symmetry}

Elasticity tensors with $D_{2}$ symmetry and positive Poisson's ratios are taken for assessing the topologies that are obtained with the procedure of Section 4 . 


\subsubsection{Studied cases}

Two composite micro-structure designs are assessed. These composites are denoted by I and II, and their effective elasticity tensors in Cartesian coordinates are:

$$
\hat{\boldsymbol{C}}^{I}=\left[\begin{array}{ccc}
0.0392 & 0.0578 & -0.0385 \\
0.0578 & 0.1050 & -0.0931 \\
-0.0385 & -0.0931 & 0.1100
\end{array}\right] \quad ; \quad \hat{\boldsymbol{C}}^{I I}=\left[\begin{array}{ccc}
0.0364 & 0.0462 & 0.0211 \\
0.0462 & 0.0785 & 0.0631 \\
0.0211 & 0.0631 & 0.0846
\end{array}\right]
$$

The same tensors in Normal coordinates, denoted $\hat{\boldsymbol{C}}_{N}^{I}$ and $\hat{\boldsymbol{C}}_{N}^{I I}$, are expressed by:

$$
\hat{\boldsymbol{C}}_{N}^{I}=\left[\begin{array}{ccc}
0.2254 & 0.0031 & 0 . \\
0.0031 & 0.0281 & 0 . \\
0 . & 0 . & 0.0006
\end{array}\right] \quad ; \quad \hat{\boldsymbol{C}}_{N}^{I I}=\left[\begin{array}{ccc}
0.1624 & 0.0043 & 0 . \\
0.0043 & 0.0363 & 0 . \\
0 . & 0 . & 0.0008
\end{array}\right]
$$

being that the rotation angles between the Cartesian and the Normal bases are $\theta_{I}=-\arctan (\sqrt{2})$ and $\theta_{I I}=\arctan (\sqrt{2})$ for materials I and II, respectively.

The composites are constituted of a stiff phase $M_{1}$ and void. Young's modulus and Poisson's ratio of the stiff phase are $E_{1}=1$. [GPa] and $\nu=0.3$, respectively. The plane stress hypothesis is assumed.

\subsubsection{The topology optimization algorithm.}

A density-based topology optimization technique combined with the Solid Isotropic Material with Penalization (SIMP) interpolation formulation ([3]) is adopted in this Section for solving the problem (2). The resulting formulation is iteratively solved with the IPOPT interior point primal-dual algorithm, see Wächter and Biegler [40] and the bibliography cited therein. The coupling of these procedures has been analyzed by Rojas-Labanda et al. [41]. Therefore, in Appendix B, we sketch a brief description of this approach remarking the filters that are used to solve these cases. Algorithm parameters: Similar to the previous examples, we use a density filter that forces the optimum solution to display a material topology with only one length scale and a Heaviside projection filter to alleviate the issue related to the presence of gray material.

The density filter described in Appendix B.2, uses a value $R=\alpha L$, where $L$ is the cell size and $\alpha$ (with $0<\alpha \leq 1$ ) is the adimensional parameter scaling the filter radius. We adopt $\alpha=0.01$. The gray material presence is diminished by using the Heaviside filter described in Appendix B.3, also discussed in Wang et al. [37]. The parameter $\beta$ handles the Heaviside filter. An external loop of the IPOPT algorithm increases the $\beta$-parameter according to the sequence $\{0,1,2,4,8, \ldots\}$. Finite element meshes with 40000 elements are used; SIMP density exponent $p=3.5$. All the 
solutions satisfy the normalized constraint of the problem (2) with the tight tolerance $(t o l=1 . e-4)$ defined in the IPOPT algorithm.

\subsubsection{Performance assessment of the design procedure}

\section{Micro-structures designed by enforcing the rules 1 and 4}

Squares cells are used and the IPOPT algorithm is initialized with two different configurations: a stiff material density randomly distributed or uniformly distributed (with initial density $\rho=0.5$ ). Additionally, two procedures are followed to solve four topology optimization problems:

i) The first procedure performs the inverse design of composites $I$ and $I I$ with the target tensors $\hat{\boldsymbol{C}}^{I}$ and $\hat{\boldsymbol{C}}^{I I}$ in Cartesian coordinates without imposing symmetries.

ii) In the second procedure, tensors $\hat{\boldsymbol{C}}^{I}$ and $\hat{\boldsymbol{C}}^{I I}$ are first rotated to Normal coordinates. Therefore, the inverse designs are performed with the target tensors $\hat{\boldsymbol{C}}_{N}^{I}$ and $\hat{\boldsymbol{C}}_{N}^{I I}$ and the Voronoi cells are aligned with their Normal basis. Furthermore, a symmetric material distribution consistent with a $p 2 \mathrm{~mm}$ plane group is imposed on the SIMP methodology. The horizontal and vertical central lines of the cells are symmetry lines. In summary, the micro-structure design is performed according to the two rules 1 and 4 describe in Section 4.

The solutions of these four cases, with the two initial configurations, are compared in Figure 12. We observe that the solutions, whose cells are aligned with the Normal basis of the target tensors and with the imposed symmetry being consistent with a $p 2 \mathrm{~mm}$ plane group (second and fourth column of the Figure), display simpler topologies. These solutions are similar to laminates which can be easily parametrized. Alternatively, the micro-architectures designed with the elasticity tensor in the original Cartesian basis display more complex topologies which result as a consequence of using square cells and periodic boundary conditions not aligned with the symmetry lines of the materials.

It is additionally noted that the solutions in Figure 12 are not very sensitive to the initial configuration taken to start the IPOPT algorithm. Even when this conclusion will be partially relativized in the following numerical tests, it is in the same line with observations reported in the literature, see Rojas-Labanda et al. [41].

\section{Micro-structures designed by enforcing the four rules}

The same composites $I$ and $I I$, with target elasticity tensor $\hat{\boldsymbol{C}}^{I}$ and $\hat{\boldsymbol{C}}^{I I}$, are next designed by following the full set of rules described in Section 4. The Voronoi cells compatible with a target elasticity displaying $D_{2}$ symmetry corresponds to $R_{p}$ or $R_{c}$ Bravais lattices. The cell slendernesses, i.e., the aspect ratio between the larger and 


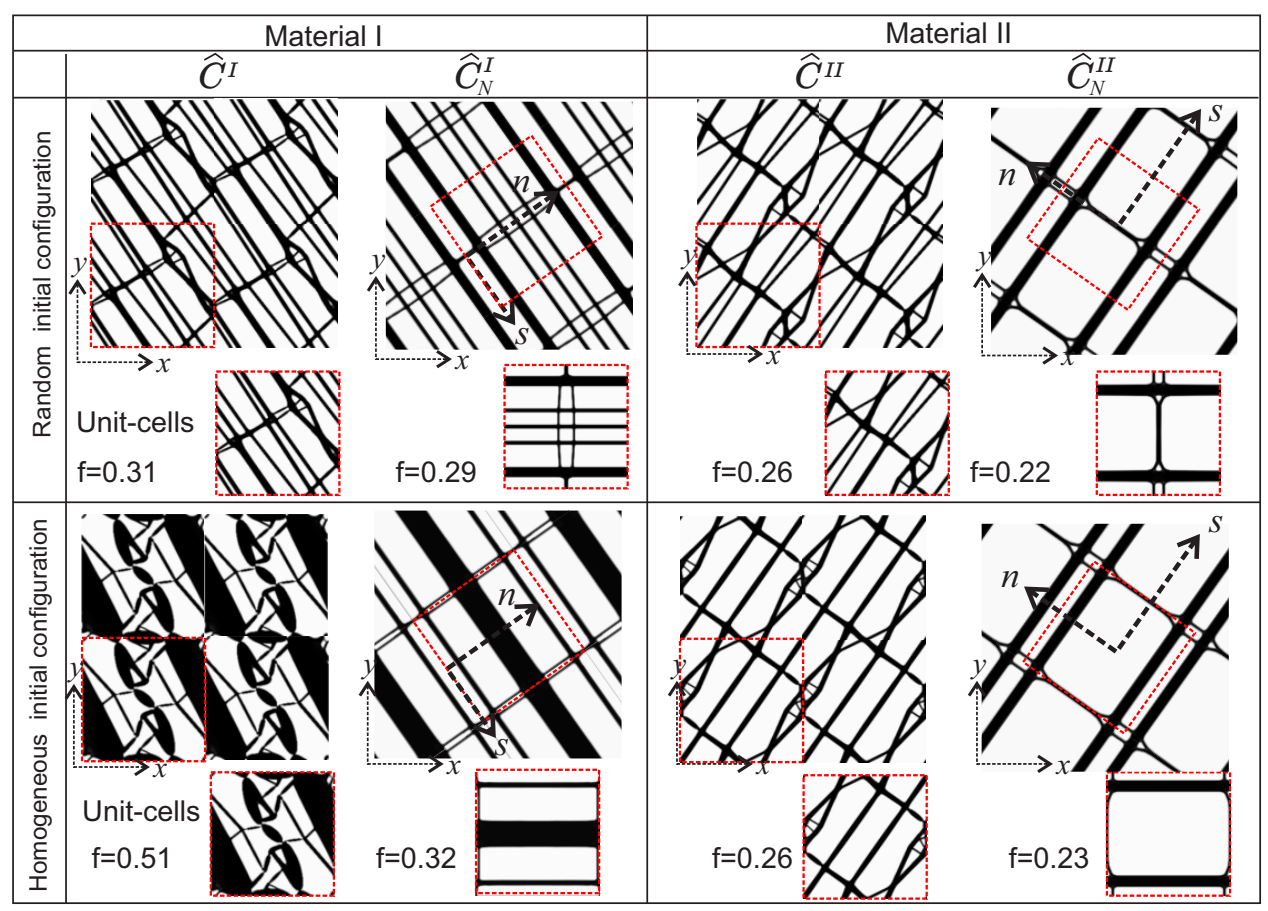

Figure 12: Four examples of micro-architecture designs. Target tensors are: $\hat{\boldsymbol{C}}^{I}$ and $\hat{\boldsymbol{C}}_{N}^{I}$ for material I; $\hat{\boldsymbol{C}}^{I I}$ and $\hat{\boldsymbol{C}}_{N}^{I I}$ for material II. Pictures correspond to the assembled micro-architectures in a direction agreeing with the Cartesian basis. Unit cells and volume fractions are also shown. 
shorter size of the cells, are assessed through the database and the criterion defined by expression (6). The shape of the cells for material I and II determined with this criterion are the rectangular cells, compatible with $R_{p}$ Bravais lattices, shown in Figure 13-a. Furthermore, an adequate plane group determining the material distribution geometry would be one of the seven plane groups denoted in Table 2 by $p m, p g, c m, p 2 m m, p 2 m g, p 2 g g$ or $c 2 m m$. In particular, we choose the plane group $p 2 m m$.

Figure 13-b depicts the topologies computed with this procedure. The effect of taking an initial configuration given by the database (topologies shown in the third column of these results) is evaluated by comparing the micro-architectures solved with different initial configurations, a stiff material density randomly distributed or uniformly distributed (with density $\rho=0.5$ ), such as shown in the first and second columns.

It can be seen that the final micro-architecture, obtained with the initial configuration taken from the database, is the simpler one constituted by bars of (almost) uniform thickness, but different length ratios. The bar lengths typically agree with the cell slenderness.

Figure 14 show the micro-architectures of material I and II that have been obtained by using SIMP with the initial configuration taken from the database.

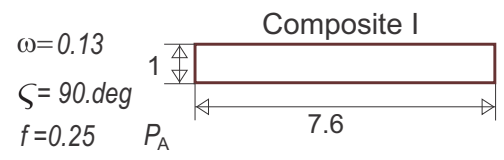

(a)

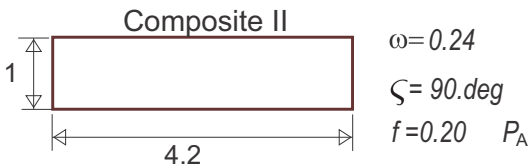

\begin{tabular}{|l|c|c|c|}
\hline & $\begin{array}{c}\text { Inital configuration: } \\
\text { random density }\end{array}$ & $\begin{array}{c}\text { Inital configuration: } \\
\text { uniform density }\end{array}$ & $\begin{array}{c}\text { Inital configuration: } \\
\text { taken from database }\end{array}$ \\
\hline$\widehat{C}_{N}^{I}$ & - & & \\
\hline$\widehat{C}_{N}^{I I}$ & & & \\
\hline
\end{tabular}

(b)

Figure 13: Micro-structure designs of composites I and II. The topology optimization problem uses the four rules of Section 4. a) Voronoi cells determined with criterion (6); b) Unit-cell solutions of the topology optimization algorithm by adopting three different initial material configurations.

\subsubsection{Discussion of results}

The obtained solutions show that enforcing periodic boundary condition along directions not aligned with the Normal basis may be the cause of attaining microstructures with complex topologies if compared with solutions obtained by follow- 


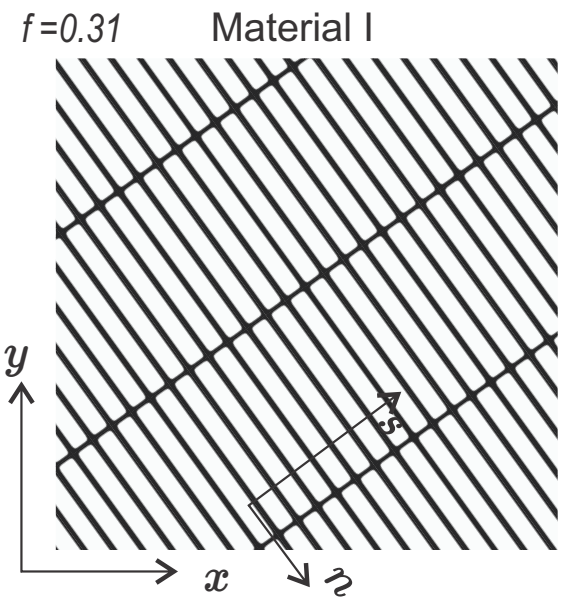

(a)

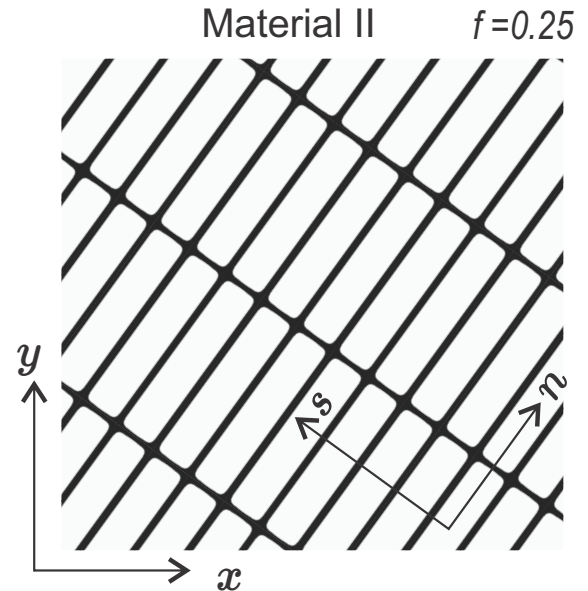

(b)

Figure 14: Micro-structure asasamblages of the composites I and II. The topology optimization problem uses the four rules of Section 4. Initial configurations are taken from the database. The resulting volume fractions are $f=0.31$ and $f=0.25$ for composites I and II, respectively.

ing the first rule of the procedure here proposed. Even simpler topologies can be attained when the initial configuration of the iterative algorithm are taken from the database. Nevertheless, a simple configuration may not be the global optimum solution of the problem.

In a similar way, the sensitivity of the attained material configuration with different design domains is evidenced in these tests. Notably, the aspect ratios of the cells also play an important role in obtaining different topology types.

\subsection{Pentamode material design}

According to Norris [42], pentamode materials are useful for constructing acoustic cloaking devices. A Pentamode material is a class of extremal material having five easy (compliant) modes of deformation in a three-dimensional space, and having only one non-easy (hard) mode of deformation ${ }^{10}$. The elasticity tensor of this material has one non-null eigenvalue and five null eigenvalues (hence the name of pentamode given to this class of material). In 1995, Milton and Cherkaev [43] have coined the

\footnotetext{
${ }^{10}$ In the following, we preserve the name of pentamode material for plane (two-dimensional) problems. Removing the third dimension and the out-of-plane field components, the elasticity tensor has only three eigenvalues. Particularizing the same concept of pentamode material, two of these eigenvalues are related to compliant modes of deformation and only one is related to a hard mode. Strictly, this material should be called bi-mode material. Additional discussion about bi-mode materials can be found in [23].
} 
name of pentamode materials in the context of linear elasticity. In the same year, Sigmund [2] has independently introduced it in the context of inverse homogenization problems.

Pentamode materials are a special class of linear anisotropic elastic solids. They can be characterized through elasticity tensors represented by:

$$
\boldsymbol{C}^{*}=\kappa^{*} \boldsymbol{S} \otimes \boldsymbol{S} ;
$$

where $\kappa^{*}$ is a pseudo-bulk modulus with the dimensions of stress and $\boldsymbol{S}$ is an adimensional symmetric second order tensor with norm not necessarily equal to one. As usual, the symbol $\otimes$ denotes the tensorial product.

The micro-structure design of a pentamode material proposed for constructing an acoustic cloaking device is here studied.

The goal of this assessment is to show that different design domains $\Omega_{\mu}$ provide markedly dissimilar solutions of the topology optimization algorithm and that the solution obtained with the systematic procedure of Section 4 display the simpler topologies.

\subsubsection{Studied cases}

Norris [42] has reported that an acoustic cloaking device can be realized by designing a layered graded pentamode material whose effective elastic properties can be determined with the analytical results presented by Gokhale et al. [44].

The design and realization of the idealized layer have been addressed in a number of works. In particular, the reported solution by Méndez et al. [39] divides the layer into 20 sub-layers whose micro-structures are then determined by means of an inverse homogenization design. The layered composite is constituted of aluminum, with a bulk modulus $\kappa_{A l}=70 . G P a$ and shear modulus $G_{A l}=25.5 G P a$, and a flexible polymer foam characterized by an isotropic material whose elastic modulus has a contrast factor $\gamma=0.00001$ times the elastic modulus of the aluminum.

Sub-layer number 15 of the acoustic cloaking device described in Méndez et al. is chosen to perform this study. Here, we only consider a partial aspect of the total layer design for the mentioned device, i.e., the micro-architecture design is only addressed without enforcing the required density constraint.

For this sub-layer in particular, the target elastic properties are characterized by the elasticity tensor described in Normal coordinates as follows:

$$
\hat{C}_{N}=\left[\begin{array}{ccc}
5.893 & 2.250 & 0 . \\
2.250 & 0.8590 & 0 . \\
0 . & 0 . & 0 .
\end{array}\right] \text {. }
$$

This tensor corresponds to a pentamode material with $D_{2}$ symmetry. The composite is designed to copy these elastic properties. 


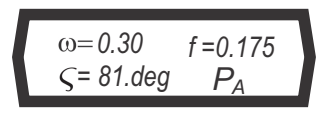

(a)

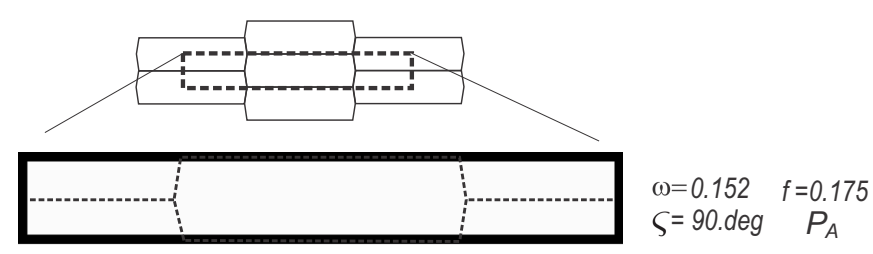

(b)

Figure 15: Pentamode material design. a) Hexagonal cell taken from the database using the criteria (6). b) Rectangular cell being compatible with the Bravais lattice whose Voronoi cell is the hexagonal cell in picture a).

\subsubsection{Topology optimization algorithm}

The level-set algorithm presented in the previous sub-Section is used in this case to solve problem (2). Similar to the technique adopted in the previous examples, we use a spatial filter to avoid topology designs with multiple length scales.

\subsubsection{Obtained results}

First, we remark that it has not been possible to attain acceptable results with square cells and the adopted mesh resolution. Therefore, the analysis is restricted to rectangular and hexagonal cells $\left(R_{p}\right.$ or $R_{c}$ Bravais lattices)which are compatible with the $D_{2}$ symmetry of the target tensor. Furthermore, the solved topologies are enforced to satisfy the $p 2 \mathrm{~mm}$ plane group.

Case A: The hexagonal cell shown in Figure 15-a has been determined with the criterion (6). The resulting parameters are: $\omega=0.3, \varsigma=81$.deg, pattern $P_{A}$ and $f=0.175$. Taking a domain of analysis defined by this hexagonal cell, we test two sub-cases, A1 and A.2, which are defined with different initial stiff phase configurations, as follows.

A.1 The initial stiff phase configuration is the one given by the solution of problem (6), such as shown in Figure 15-a.

A.2 The initial configuration consists of a random stiff phase distribution. Five different instances of this sub-case are solved.

Case B: the rectangular cell shown in Figure 15-b is taken as the design domain. This cell with parameters $\omega=0.1517, \varsigma=90 . \mathrm{deg}$ can reproduce a similar microstructure as that given by the hexagonal cell of the case A. The three sub-cases are solved with the following initial conditions:

B.1 stiff bars are disposed in the boundary of the cell; 


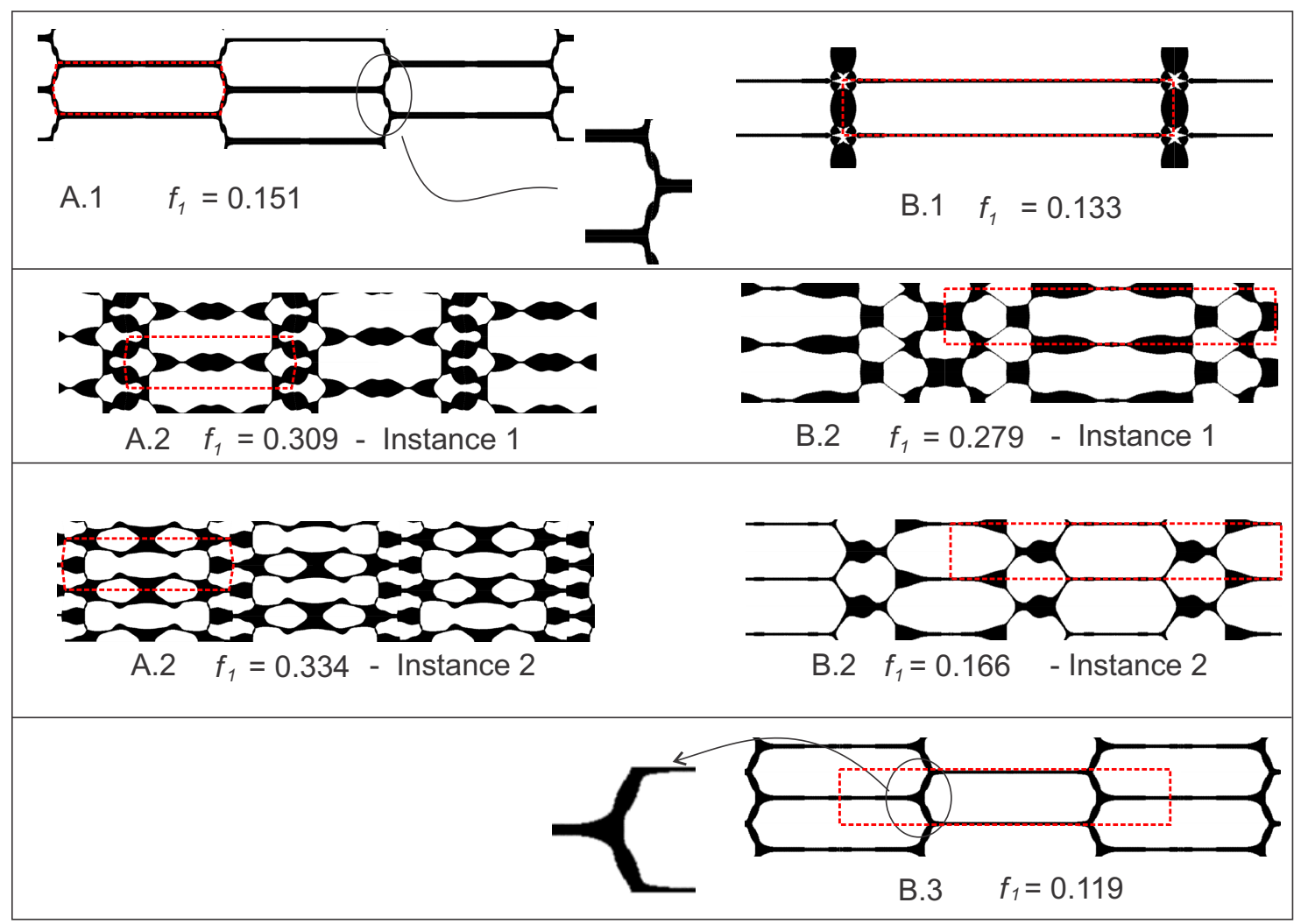

Figure 16: Pentamode material designs. Topologies attained with sub-cases A1, A.2 and $\mathrm{B}$. The volume fraction of each case is $f_{1}$. 


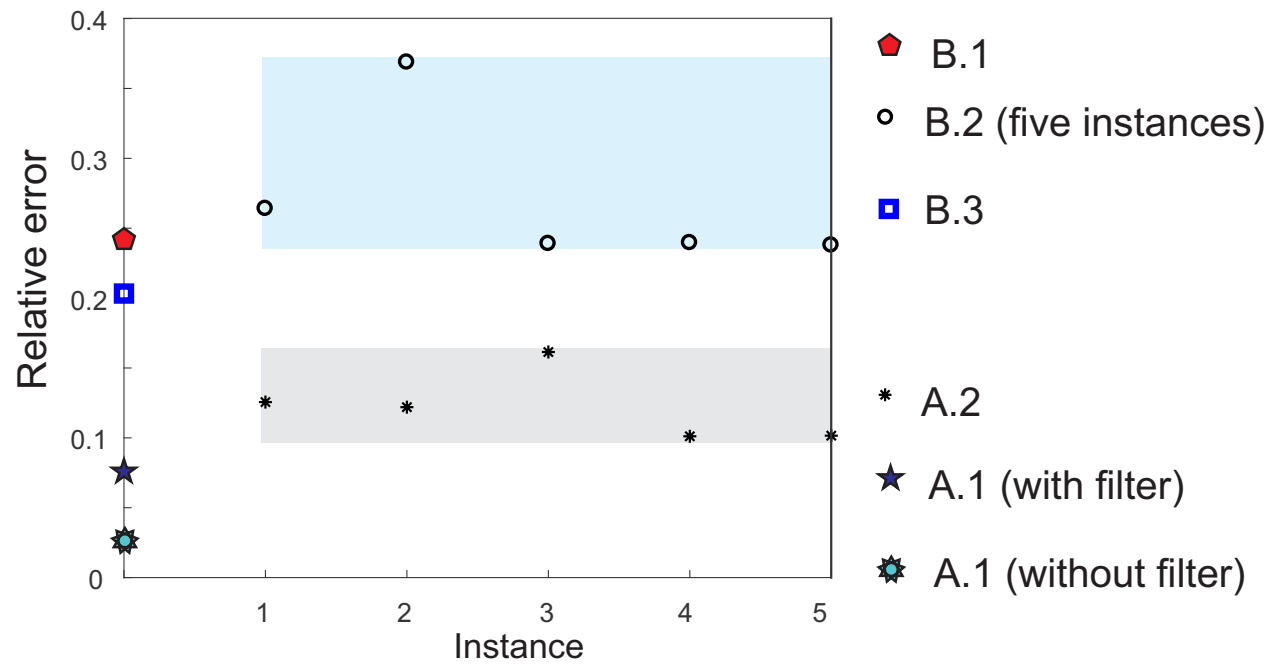

Figure 17: Pentamode material design. Relative errors $\left\|\boldsymbol{C}^{h}-\hat{\boldsymbol{C}}\right\| /\|\hat{\boldsymbol{C}}\|$ computed for the solutions obtained with the Level-set algorithm.

B.2 a random distribution of stiff phase. Five instances are solved with initial random configurations.

B.3 stiff bars are disposed in a hexagonal array. This hexagonal array is the same as that of the initial configuration of Case A.1, but here, it is projected onto the rectangular cell such as shown in Figure 15-b.

The topologies obtained in all these cases are shown in Figure 16.

Figure 17 plots the relative errors $\left\|\boldsymbol{C}^{h}-\hat{\boldsymbol{C}}\right\| /\|\hat{\boldsymbol{C}}\|$ for all the tested cases solved with the Level-set algorithm. The solution with the lower error is computed under the following conditions: the hexagonal cell and the initial configuration are taken from the database, and the algorithm is run without the filter. In the remaining cases, the filter penalizes the solutions to attain effective elasticity tensors closer to the target one. In any case, it is notable that the hexagonal cell provides more accurate solutions if compared with those obtained, in comparable circumstances, with the rectangular cell.

\section{Conclusions}

The associations presented in Tables 2, 3, 4 and Figure 6 constitute the fundamental ingredients introduced in this paper to connect the symmetries characterizing the material configurations and the physical properties. They also constitute the basic components to identify an adequate micro-architecture design procedure of composites whose effective properties fulfill a given elastic response. 
Based on these notions, four rules have been proposed in this work. These rules define a systematic procedure that can be followed to facilitate the micro-structure design carried out by means of an inverse homogenization technique.

Through a number of numerical assessments, it has been shown that the conventional square cells, adopted as the design domain for the inverse homogenization techniques formulated as a topology optimization problem, are not adequate to obtain simple micro-architecture configurations in general situations. Instead, appropriate cells and plane groups, identifying the material configuration symmetry within the cell, can alternatively be chosen to guarantee the attainment of composites with simple topologies and effective elastic responses displaying identical symmetries to the target ones. These conclusions are independent of which topology optimization algorithm is taken to solve the inverse homogenization problem.

An observation here remarked is that the proposed rules could be extended to inverse homogenization techniques involving 3D elastic material designs. The generalization of the concepts involving crystal physics and the related symmetries in 3D could provide some hints to conceive base cells for more general domain designs in $3 \mathrm{D}$ problems.

\section{Acknowledgments}

The authors acknowledge the financial support from CONICET and ANPCyT (grants PIP 2013-2015 631 and PICT 2014-3372 and 2016-2673).

\section{A APPENDIX: Solving the topology optimiza- tion problem with Topological Derivate Algo- rithm}

The topology optimization problem (2) can be solved by introducing a smooth level set-funtion, $\psi \in C^{0}\left(\Omega_{\mu}\right)$, satisfying

$$
\psi(\boldsymbol{y})= \begin{cases}<0 & \forall \boldsymbol{y} \in \Omega_{\mu}^{2} \\ >0 & \forall \boldsymbol{y} \in \Omega_{\mu}^{1} \\ 0 & \text { in the interfaces }\end{cases}
$$

and utilizing an augmented Lagrangian technique, see Lopes et al. [45]. In this case, the problem is rewritten as follows

$$
\max _{\lambda} \min _{\psi} \mathcal{T}(\psi, \lambda)
$$


with:

$$
\mathcal{T}(\psi, \lambda)=\int_{\Omega_{\mu}} \chi(\psi) d \Omega+\lambda\left(\left\|\boldsymbol{C}^{h}(\psi)-\hat{\boldsymbol{C}}\right\|\right)+\frac{\alpha}{2}\left(\left\|\boldsymbol{C}^{h}(\psi)-\hat{\boldsymbol{C}}\right\|\right)^{2}
$$

where $\lambda$ is the Lagrange multiplier and $\alpha$ is the penalty parameter of the augmented term.

The algorithm for solving the problem (17) utilizes two nested loops. In an internal loop, the objective function $\mathcal{T}$ is minimized by holding fixed $\lambda$ and $\alpha$. This loop, with index denoted $k$, consists of a level-set function-based iteration. While an external loop, with index denoted $l$, modifies iteratively $\lambda$.

The minimum of $\mathcal{T}$ in the internal loop is searched with a descent direction algorithm. For problem (17), the topological derivative used to estimate the descent direction is given by

$$
D_{\psi} \mathcal{T}(\psi, \lambda)=1-\left(\left(\lambda-\alpha\left\|\boldsymbol{C}^{h}-\hat{\boldsymbol{C}}\right\|\right) \frac{\left(\boldsymbol{C}^{h}-\hat{\boldsymbol{C}}\right): D_{\psi} \boldsymbol{C}^{h}}{\left\|\boldsymbol{C}^{h}-\hat{\boldsymbol{C}}\right\|}\right)
$$

where $D_{\psi} \boldsymbol{C}^{h}$ is the topological derivative of the homogenized elasticity tensor, see [5] for an additional description of this term. Then, we define the function :

$$
g(\boldsymbol{y})=\left\{\begin{array}{ll}
-\left(D_{\psi} \mathcal{T}\right) & \text { if }: \psi<0 \\
+\left(D_{\psi} \mathcal{T}\right) & \text { if }: \psi>0
\end{array},\right.
$$

The updating formula for $\psi$, at the $(k+1)$-th internal loop, is defined by

$$
\psi^{k+1}=\psi^{k}+\tau g,
$$

with the scaling factor $\tau$ being determined by means of a line search technique.

In the $(l+1)$-th external loop, the Lagrange multiplier $\lambda$ is updated using the Uzawa algorithm

$$
\lambda^{l+1}=\max \left(0, \lambda^{l}+\alpha\left\|\boldsymbol{C}^{h}-\hat{\boldsymbol{C}}\right\|\right) .
$$

The penalty parameter $\alpha$ is hold fixed during the full process.

A local optimality criterion of problem (17), see Amstutz [46], is given by the condition

$$
D_{\psi} \mathcal{T}>0 \quad ; \quad \forall \boldsymbol{y} \in \Omega_{\mu}
$$

which can be implemented by verifying the inequality

$$
\left[\frac{\int_{\Omega_{\mu}} g \psi d V}{\|g\|_{L^{2}}\|\psi\|_{L^{2}}}\right]>\left(1-\operatorname{tol}_{\psi}\right)
$$

combined with

$$
\left\|\boldsymbol{C}^{h}-\hat{\boldsymbol{C}}\right\|<\operatorname{tol}_{C}
$$


Additionally, a Helmoltz-type filter taken from [36] is implemented. The smooth level set function $\tilde{\psi}$ in each iteration $(k+1)$-iteration is computed by solving the field equation:

$$
r_{l s}^{2} \nabla^{2} \tilde{\psi}^{k+1}+\tilde{\psi}^{k+1}=\psi^{k+1}
$$

with homogeneous boundary conditions $d\left(\tilde{\psi}^{k+1}\right) / d \boldsymbol{n}=0$ on the boundary of $\Omega_{\mu}$. The filter measure $r_{l s}$ defines the minimum length scale in the topology optimization problem.

\section{B APPENDIX: Solving the topology optimiza- tion problem with SIMP}

The SIMP technique reported in Bendsoe and Sigmund [3] is here adopted. In this technique, the design domain $\Omega_{\mu}$ is subdivided using a finite element mesh, and each element $e$ is assigned a density $\rho^{e}$. This density represents the presence of soft or stiff materials (zero density for soft phase, unit density for stiff phase) by defining a non-linear interpolation of the elasticity tensors, $\boldsymbol{C}_{2}$ for soft and $\boldsymbol{C}_{1}$ for stiff phases, as follows:

$$
\boldsymbol{C}^{e}\left(\rho^{e}\right)=\boldsymbol{C}_{2}+\left(\rho^{e}\right)^{p}\left(\boldsymbol{C}_{1}-\boldsymbol{C}_{2}\right) .
$$

This is a smooth transition of elastic properties with density varying in the interval $0 \leq \rho^{e} \leq 1$. The intermediate densities $\left(0<\rho^{e}<1\right)$ are penalized by defining the power law of $\rho$ with exponent $p>1$.

Given the distribution of densities in $\Omega_{\mu}$ and the interpolated elasticity tensors (27), the homogenized elasticity tensor, $\boldsymbol{C}^{h}$ can be computed through standard procedures. This notation: $\boldsymbol{C}^{h}(\rho)$, remarks the dependence of $\boldsymbol{C}^{h}$ with the spatial distribution of the so-defined densities.

\section{B.1 Topology optimization problem}

The inverse homogenization technique can be formulated as follows:

$$
\begin{array}{r}
\min _{\rho} \int_{\Omega_{\mu}} \rho d \Omega, \\
\text { such that: }\left\|\boldsymbol{C}^{h}(\rho)-\hat{\boldsymbol{C}}\right\|=0 .
\end{array}
$$

\section{B.2 Density filter}

The density filter taken from [36] is also implemented in the context of the SIMP methodology. The density of an element $\rho^{e}$ is taken as being the weighted average of the design variables of the neighbor finite elements. The neighborhood is defined 
as a circle in $2 \mathrm{D}$ with the radius $\mathrm{R}$. Then, the application of a density filter leads to the variables $\bar{\rho}^{e}$ :

$$
\bar{\rho}^{e}=\frac{\sum_{i=1}^{Q} w_{i}^{e} \Omega_{i} \rho_{i}}{\sum_{i=1}^{Q} r_{i}^{e} \rho_{i}}
$$

where $Q$ is the number of elements in the neighborhood of element $e$ defined by $R$, $r_{i}^{e}$ is the distance from element $i$ to elements $e, \Omega_{i}$ is the volume of element $i, w_{i}^{e}$ is a weighting factor defined as:

$$
w_{i}^{e}=\max \left(0, R-r_{i}^{e}\right)
$$

\section{B.3 Volume preserving Heaviside filter}

The filtered variables $\bar{\rho}^{e}$ are further transformed into element densities $\hat{\bar{\rho}}^{e}$ by means of an additional Heaviside filter: values smaller than a threshold value $\mathrm{g}$ are projected to 0 ; values larger than $\eta$ are projected to 1 . In this work it is used a Volume preserving Heaviside filter ([47]).

$$
\hat{\bar{\rho}}^{e}= \begin{cases}\eta\left[e^{-\beta\left(1-\bar{\rho}^{e} / \eta\right)}-\left(1-\bar{\rho}^{e} / \eta\right) e^{-\beta}\right] & 0 \leq \bar{\rho}^{e} \leq \eta \\ (1-\eta)\left[1-e^{-\beta\left(\bar{\rho}^{e}-\eta\right) /(1-\eta)}\right. & , \\ +\left(\bar{\rho}^{e}-\eta\right) e^{-\beta} /(1-\eta) & \eta<\bar{\rho}^{e} \leq 1\end{cases}
$$

where $\beta$ is a parameter taken as $\beta \geq 1$. When the Heaviside filter is applied in each iteration, the volume fraction is preserved by satisfying the following equation:

$$
\sum_{i=1}^{N} \rho_{i} \Omega_{i}=\sum_{i=1}^{N} \hat{\bar{\rho}}_{i}(\eta) \Omega_{i} .
$$

where, the left-hand side is the volume before the filtering, and the right-hand side is the volume after applying the filter. This equation is satisfied by adjusting the parameter $\eta$ with a bisection iterative method. The number of elements is $N$.

Finally, the objective function and the homogenized elasticity tensor $\boldsymbol{C}^{h}$ in problem (28) are computed with the filtered density $\hat{\bar{\rho}}^{e}$.

\section{B.4 Procedure for imposing the plane group symmetry on the micro-architecture topology}

The design variable are updated at the end of each iteration of the topology optimization algorithm to satisfy the symmetry requirement specified by the plane group. For the level set methodology, the design variable update is computed according to the following sequence of operations. Initially, the set of points of the 
spatial domain related through the space group symmetry operations are found. One node of this set is chosen as the master one, and the remaining are the slave ones. At each iteration: 1) calculate element sensitivities and project to nodes; 2) compute the averaging of sensitivities corresponding to nodes that are linked through the symmetry operations, 3) using equation (20), update the level-set variable only for the master nodes and 4) copy the value of the master node level-set variable to all the slave nodes. For the SIMP methodology, the updating procedure is the following: 1) calculate sensitivities for all elements; 2) identifying "master elements" or design variables, 3) compute the averaging of sensitivities that are linked through the symmetry operations, 4) solve the optimization update only for the reduced set of master elements, 5) copy the master design variable value to the linked elements.

\section{References}

[1] O. Sigmund. Materials with prescribed constitutive parameters: an inverse homogenization problem. International Journal of Solids and Structures, 31 (17):2313-2329, 1994.

[2] O. Sigmund. Tailoring materials with prescribed elastic properties. Mechanics of Materials, 20(4):351-368, 1995.

[3] M.P. Bendsoe and O. Sigmund. Topology optimization: theory, methods, and applications. Springer Science \& Business Media, 2003.

[4] X. Huang, S.W. Zhou, Y.M. Xie, and Q. Li. Topology optimization of microstructures of cellular materials and composites for macrostructures. Computational Materials Science, 67:397-407, 2013.

[5] S. Amstutz, S.M. Giusti, A.A. Novotny, and E.A. de Souza Neto. Topological derivative for multi-scale linear elasticity models applied to the synthesis of microstructures. International Journal for Numerical Methods in Engineering, 84(6):733-756, 2010.

[6] A Ferrer, J Oliver, JC Cante, and O Lloberas-Valls. Vademecum-based approach to multi-scale topological material design. Advanced Modeling and Simulation in Engineering Sciences, 3(1):23, 2016.

[7] G. H. Paulino, E.C.N. Silva, and Chau H. Le. Optimal design of periodic functionally graded composites with prescribed properties. Structural and Multidisciplinary Optimization, 38(5):469-489, 2009.

[8] P.G. Coelho, L.D. Amiano, J.M. Guedes, and H.C. Rodrigues. Scale-size effects analysis of optimal periodic material microstructures designed by the inverse homogenization method. Computers \& Structures, 174:21-32, 2016. 
[9] A.R. Diaz and A. Benard. Designing materials with prescribed elastic properties using polygonal cells. International Journal for Numerical Methods in Engineering, 57(3):301-314, 2003.

[10] O. Sigmund. A new class of extremal composites. Journal of the Mechanics and Physics of Solids, 48(2):397-428, 2000.

[11] C. Barbarosie, D.A. Tortorelli, and S. Watts. On domain symmetry and its use in homogenization. Computer Methods in Applied Mechanics and Engineering, 320:1-45, 2017.

[12] D. Lukkassen, A. Meidell, and S. Vigdergauz. On the elastic deformation of symmetric periodic structures. The Quarterly Journal of Mechanics and Applied Mathematics, 56(3):441-454, 2003.

[13] J.F. Nye. Physical Properties of Crystals: Theri representation be tensors and matrices, volume 146. Clarendon Press- Oxford, 2006.

[14] Q.S. Zheng and J.P. Boehler. The description, classification, and reality of material and physical symmetries. Acta Mechanica, 102(1-4):73-89, 1994.

[15] M.P. Bendsøe and N. Kikuchi. Generating optimal topologies in structural design using a homogenization method. Computer methods in applied mechanics and engineering, 71(2):197-224, 1988.

[16] F. Feyel and J.L. Chaboche. Fe2 multiscale approach for modelling the elastoviscoplastic behaviour of long fibre sic/ti composite materials. Computer methods in applied mechanics and engineering, 183(3-4):309-330, 2000.

[17] J.C. Michel, H. Moulinec, and P Suquet. Effective properties of composite materials with periodic microstructure: a computational approach. Computer methods in applied mechanics and engineering, 172(1-4):109-143, 1999.

[18] J. Sólyom. Fundamentals of the Physics of Solids: Volume 1: Structure and Dynamics, volume 1. Springer Science \& Business Media, 2007.

[19] B. Souvignier. A general introduction to space groups. International Tables for Crystallography, A:22-41, 2016. Chapter:1.3.

[20] K. Landwehr. Visual discrimination of the 17 plane symmetry groups. Symmetry, 3(2):207-219, 2011.

[21] T.C. Ting. Anisotropic elasticity: theory and applications. Number 45. Oxford University Press, 1996. 
[22] N. Auffray and P. Ropars. Invariant-based reconstruction of bidimensional elasticity tensors. International Journal of Solids and Structures, 87:183-193, 2016.

[23] JM Podestá, CG Méndez, S Toro, Alfredo Edmundo Huespe, and J Oliver. Material design of elastic structures using voronoi cells. International Journal for Numerical Methods in Engineering, 115(3):269-292, 2018.

[24] E. Andreassen, B.S. Lazarov, and O. Sigmund. Design of manufacturable 3d extremal elastic microstructure. Mechanics of Materials, 69(1):1-10, 2014.

[25] M.G. Rashed, M. Ashraf, R.A.W. Mines, and P.J. Hazell. Metallic microlattice materials: A current state of the art on manufacturing, mechanical properties and applications. Materials \& Design, 95:518-533, 2016.

[26] Y. Wang, L. Zhang, S. Daynes, H. Zhang, S. Feih, and M.Y. Wang. Design of graded lattice structure with optimized mesostructures for additive manufacturing. Materials \&5 Design, 2018.

[27] H. M.A. Kolken and A.A. Zadpoor. Auxetic mechanical metamaterials. $R S C$ Advances, 7(9):5111-5129, 2017.

[28] Y. Jiang and Y. Li. 3d printed auxetic mechanical metamaterial with chiral cells and re-entrant cores. Scientific reports, 8(1):2397, 2018.

[29] J.M. Podestá, S. Toro, C. Méndez, and Huespe. Homogenized elasticity tensor database of periodic composites with microarchitectures displaying symmetric topologies, 2018. Dataset available from Mendeley, http://dx.doi.org/10.17632/7kgf9xnrhb.1.

[30] A.V. Cherkaev and L.V. Gibiansky. Coupled estimates for the bulk and shear moduli of a two-dimensional isotropic elastic composite. Journal of the Mechanics and Physics of Solids, 41(5):937-980, 1993.

[31] X. Ren, R. Das, P. Tran, T.D. Ngo, and Y.M. Xie. Auxetic metamaterials and structures: A review. Smart Materials and Structures, 2018.

[32] U.D. Larsen, O. Signund, and S. Bouwsta. Design and fabrication of compliant micromechanisms and structures with negative poisson's ratio. Journal of Microelectromechanical Systems, 6(2):99-106, 1997.

[33] L. Cabras and M. Brun. Auxetic two-dimensional lattices with poisson's ratio arbitrarily close to -1. In Proceedings of the Royal Society of London A: Mathematical, Physical and Engineering Sciences, volume 470, pages 1-23, 2014. 
[34] K.E. Evans and A.W Alderson. Auxetic materials: functional materials and structures from lateral thinking! Advanced materials, 12(9):617-628, 2000.

[35] S. Amstutz and H. Andrä. A new algorithm for topology optimization using a level-set method. Journal of Computational Physics, 216(2):573-588, 2006.

[36] B. S. Lazarov and O. Sigmund. Filters in topology optimization based on helmholtz-type differential equations. Int. J. Numer. Meth. Engng, 86:765-781, 2011.

[37] F. Wang, B. S. Lazarov, and O. Sigmund. On projection methods, convergence and robust formulations in topology optimization. Structural and Multidisciplinary Optimization, 43(6):767-784, 2011.

[38] S. Meille and E.J. Garboczi. Linear elastic properties of 2d and 3d models of porous materials made from elongated objects. Modelling and Simulation in Materials Science and Engineering, 9(5):371, 2001.

[39] C. Méndez, J.M. Podestá, O. Lloberas-Valls, S. Toro, A.E. Huespe, and J. Oliver. Computational material design for acoustic cloaking. International Journal for Numerical Methods in Engineering, 2017.

[40] A. Wächter and L.T. Biegler. On the implementation of a primal-dual interior point filter line search algorithm for large-scale nonlinear programming. Mathematical Programming, 106(1):25-57, 2006.

[41] S. Rojas-Labanda, O. Sigmund, and M. Stolpe. A short numerical study on the optimization methods influence on topology optimization. Structural and Multidisciplinary Optimization, 56(6):1603-1612, 2017.

[42] A.N. Norris. Acoustic cloaking theory. In Proceedings of the Royal Society of London A: Mathematical, Physical and Engineering Sciences, volume 464, pages 2411-2434, 2008.

[43] G.W. Milton and A.V. Cherkaev. Which elasticity tensors are realizable? Journal of engineering materials and technology, 117(4):483-493, 1995.

[44] N.H. Gokhale, J.L. Cipolla, and A.N. Norris. Special transformations for pentamode acoustic cloaking. The Journal of the Acoustical Society of America, 132(4):2932-2941, 2012.

[45] C.G. Lopes, R.B. dos Santos, and A.A. Novotny. Topological derivative-based topology optimization of structures subject to multiple load-cases. Latin American Journal of Solids and Structures, 12(5):834-860, 2015. 
[46] S. Amstutz. Analysis of a level set method for topology optimization. Optimization Methods and Software, 26(4-5):555-573, 2011.

[47] S. Xu, Y. Cai, and G. Cheng. Volume preserving nonlinear density filter based on heaviside functions. Structural and Multidisciplinary Optimization, 41(4): 495-505, 2010. 